Bundesgesundheitsbl 2020 - 63:484-501 https://doi.org/10.1007/s00103-020-03118-7 Online publiziert: 25 . März 2020

(c) Springer-Verlag GmbH Deutschland, ein Teil von Springer Nature 2020

\section{Anforderungen der Hygiene an abwasserführende Systeme in medizinischen Einrichtungen}

\author{
Empfehlung der Kommission \\ für Krankenhaushygiene und \\ Infektionsprävention (KRINKO) beim Robert \\ Koch-Institut
}

\section{Inhaltsverzeichnis}

1. Einleitung

2. Geltungsbereich und Zielgruppe

3. Bezug zu vorausgegangenen Empfehlungen

4. Begriffsbestimmung abwasserführender Systeme

5. Gesetzliche Grundlage und technische Regeln für abwasserführende Systeme

6. Abwasserassoziierte Infektionsrisiken

6.1 Infektionserreger

6.2 Übertragung

6.3 Wirksamkeit abwasserassoziierter Hygienemaßnahmen

6.3.1 Nosokomiale Infektionsausbrüche

6.3.2 Vorkommen gramnegativer Infektionserreger

6.4 Einzelne Bereiche des abwasserführenden Systems

6.4.1 Waschbecken

6.4.2 Toiletten

6.4.3 Steckbecken

6.4.4 Duschen und Duschabläufe

6.4.5 Abwasserführendes Leitungssystem im Krankenhaus

6.4.6 Entleerung von Dialysebeuteln

6.4.7 Abwasserführendes System in Krankenhausküchen

7. Ökologie, Reservoire und mit abwasserführenden Systemen assoziierte Übertragung

7.1 Desinfektionswirkstoffe zur Elimination von Biofilmen

7.2 Abwasserführende Systeme als Reservoir für die Ausbreitung von Antibiotikaresistenzen

8. Empfehlungen

8.1 Aufklärung, Information, Schulung und Organisation

8.2 Design von Waschbecken, sanitären Anlagen und Duschen

8.3 Desinfektion im Sanitärbereich

8.4 Ausgussbecken in unreinen Räumen

8.5 Steckbeckenspüler

8.6 Abwasserführende Systeme

8.7 Maßnahmen bei Havariefällen von Abwasserleitungen

8.8 Küchen

8.9 Maßnahmen bei Ausbrüchen

Abkürzungsverzeichnis

Literatur

Informativer Anhang

\section{Zusatzmaterial online}

Das Zusatzmaterial online (https:// doi.org/10.1007/s00103-020-03118-7)

beinhaltet einen informativen Anhang zu der vorliegenden Empfehlung. Der Inhalt des Zusatzmaterials entspricht der Tabelle $2 \mathrm{im}$ Heft.

\section{Einleitung}

Abwassersystemen (bzw. dem darin enthaltenen Abwasser) als mögliches Reservoir für nosokomiale Infektionserreger in medizinischen Einrichtungen wurde bisher nicht die entsprechende Aufmerksamkeit gewidmet. Es gibt bisher keine gesonderten Regularien, z. B. in entsprechenden Empfehlungen der Kommission für Krankenhaushygiene und Infektionsprävention (KRINKO), die im Rahmen anderer Hygienestrategien zur Bekämpfung nosokomialer Infektionen erstellt wurden. Abwasser aus Krankenhäusern wurde hinsichtlich seiner mikrobiellen Zusammensetzung wie kommunales Abwasser eingestuft. Beide Abwasserquellen wurden als vergleichbar potenziell infektiös betrachtet. Es wurde in der Folge aus abwassertechnischen Gründen keine gesonderte hygienische Behandlung, Separierung oder getrennte Entsorgung von Körperflüssigkeiten und Ausscheidungen als notwendig erachtet.

Mittlerweile liegen jedoch insbesondere im Zusammenhang mit gramnegativen Enterobacterales und nicht-fermentierenden Bakterien (Acinetobacter spp., Pseudomonas aeruginosa, etc.), u. a. auch von 


Tab. 1 Kategorien in der Richtlinie für Krankenhaushygiene und Infektionsprävention (2010)
$\begin{array}{ll}\text { Kategorie IA } & \begin{array}{l}\text { Diese Empfehlung basiert auf gut konzipierten systematischen Reviews oder einzelnen hochwertigen randomisierten kontrollierten } \\ \text { Studien. }\end{array} \\ \text { Kategorie IB } & \begin{array}{l}\text { Diese Empfehlung basiert auf klinischen oder hochwertigen epidemiologischen Studien und strengen, plausiblen und nachvollziehba- } \\ \text { ren theoretischen Ableitungen. }\end{array} \\ \text { Kategorie II } & \begin{array}{l}\text { Diese Empfehlung basiert auf hinweisenden Studien/Untersuchungen und strengen, plausiblen und nachvollziehbaren theoretischen } \\ \text { Ableitungen. }\end{array} \\ \text { Kategorie III } & \begin{array}{l}\text { Maßnahmen, über deren Wirksamkeit nur unzureichende oder widersprüchliche Hinweise vorliegen, deshalb ist eine Empfehlung } \\ \text { nicht möglich. }\end{array} \\ \text { Kategorie IV } \quad \text { Anforderungen, Maßnahmen und Verfahrensweisen, die durch allgemein geltende Rechtsvorschriften zu beachten sind. }\end{array}$

carbapenemasebildenden und colistinresistenten Erregern, Belege für die Bedeutung abwasserführender Systeme als Reservoir für fakultativ pathogene Erreger und Ursache für u. U. jahrelang persistierende Ausbrüche im Krankenhaus vor. Dazu zählen u.a. Waschbecken, Siphon, Duschablauf und Toilette. Daher wird es als erforderlich angesehen, auf die Relevanz von Abwassersystemen als Reservoir für gramnegative Mikroorganismen hinzuweisen. Es sollen Empfehlungen bzw. Vorschläge zur Infektionsprävention einschließlich baulich-funktioneller und betrieblich-organisatorischer Maßnahmen abgeleitet und bekannt gemacht werden. Gleichzeitig sind die Erkenntnisse im Rahmen des Managements von Ausbrüchen zu berücksichtigen, sofern ein $\mathrm{Zu}$ sammenhang vermutet wird bzw. möglich erscheint. Wesentliche Publikationen finden sich im Literaturverzeichnis.

Im Darm von Patienten ${ }^{1}$ können Enterobacterales mit erworbener Antibiotikaresistenz über lange Zeit persistieren, über die Ausscheidungen ins Abwasser u.a. des patientennahen Umfeldes gelangen und hier Resistenzen austauschen bzw. neue Resistenzen auf Grund des zunehmenden Vorkommens von Antibiotika im Abwasser entwickeln. Von hier aus können diese Erreger wieder auf andere Patienten übertragen werden [1]. Es ist davon auszugehen, dass durch Unterbrechung dieses circulus vitiosus das Infektionsrisiko durch multiresistente Erreger (MRE) reduziert wird.

\footnotetext{
1 Grundsätzlich sind in diesem Dokument bei allen Berufs- bzw. Gruppenbezeichnungen immer alle Geschlechter gemeint.
}

\section{Geltungsbereich und Zielgruppe}

Die vorliegende Empfehlung richtet sich in erster Linie an Hygienefachpersonal, Ärzte und Pflegepersonal von Kliniken und anderen medizinischen Einrichtungen wie etwa Pflegeheimen sowie an Mitarbeiter des öffentlichen Gesundheitsdienstes. Sie bezieht sich auf abwasserführende Systeme in medizinischen Einrichtungen. Die Inhalte, vor allem die Beispiele für Präventionsmaßnahmen, sind auch für Verantwortliche der Haustechnik, Architekten, Umweltbehörden und Abwasserentsorger relevant.

\section{Bezug zu vorausgegangenen Empfehlungen}

Bezüge zu weiteren Empfehlungen der KRINKO ergeben sich insbesondere zu:

- den Anforderungen an die Hygiene bei der Reinigung und Desinfektion von Flächen [2];

- den Anforderungen an die Hygiene bei der medizinischen Versorgung von immunsupprimierten Patienten [3] sowie zur

- Händehygiene in Einrichtungen des Gesundheitswesens [4].

Die Definition der Kategorien, die der KRINKO-Empfehlungen zugrunde gelegt werden, ist in $\bullet$ Tab. 1 aufgeführt.

\section{Begriffsbestimmung abwasserführender Systeme}

Im Folgenden wird unter „abwasserführenden Systemen" medizinischer Einrichtungen das gesamte abwasserführende System ab der Stelle innerhalb der medizinischen Einrichtung verstanden, an der Körperflüssigkeiten und Ausscheidungen in das abwasserführende System gelangen. Somit beginnt das abwasserführende System in medizinischen Einrichtungen bereits mit:

- Waschbecken einschließlich Ablauföffnung, Siphon und ggf. Waschbeckenüberlauf;

- WC-Becken in Toiletten;

- Duschbecken einschließlich Ablauföffnung in Duschen;

- Abflüssen von Badewannen und Gebärwannen;

- Speibecken von Dentaleinheiten;

- Ausgussbecken für Abwasser unterschiedlicher Art (in der Regel in sog. unreinen Räumen);

- Steckbeckenspülern;

- Auffangsystemen für Spülwasser in urologischen Eingriffsräumen (z. B. für TUR-Eingriffe) bzw. Operationseinheiten;

- Abwasserabläufen in Küchen (Bodenablauf);

- ggf. weiteren Systemen.

Insbesondere in den initialen Bereichen des Abwassersystems wie Toilette, Bodenabläufe, Waschbecken und Duschabläufe, Ausgussbecken in unreinen Räumen sowie den Abwasseröffnungen in Küchen besteht die Möglichkeit der direkten bzw. indirekten Exposition von Patienten gegenüber Krankheitserregern. Diese kann auch das medizinische Personal, Instrumente, Geräte, Reinigungsutensilien, Desinfektionsmittelspender und Tuchspendersysteme sowie Küchenpersonal und Lebensmittel betreffen.

Ziel der Umsetzung der Anforderungen der Hygiene an abwasserführende Systeme in medizinischen Einrichtungen ist es, das Risiko einer Exposition und möglicherweise damit verbundenen nachfolgender Kontaminationen, Kolo- 
nisationen oder Infektionen als Teil eines Maßnahmenbündels zu minimieren. Dies erfolgt u.a. durch baulich-funktionelle bzw. -technische sowie betrieblich-organisatorische Maßnahmen einschließlich des Designs von wasser- und abwasserführenden Systemen (z. B. durch Aerosolverminderung) sowie durch Information an das medizinische Personal, die Haustechniker und an die Patienten. Dabei sind insbesondere bei der Entsorgung von vom Patienten stammenden kontaminierten Flüssigkeiten (z.B. Urin, Fäkalien) technische Konzeptionen zu fördern, die dem medizinischen Personal eine kontaminationsfreie Entsorgung ermöglichen.

Die nachfolgenden Empfehlungen und Maßnahmen sind mit dem zuständigen Krankenhaushygieniker abzustimmen.

\section{Gesetzliche Grundlage und technische Regeln für abwasserführende Systeme}

Gesetzliche Grundlage ist $\$ 41$ des Infektionsschutzgesetzes aus dem Jahr 2000 [5]. Hierin heißt es:

1. Die Abwasserbeseitigungspflichtigen haben darauf hinzuwirken, dass $A b$ wasser so beseitigt wird, dass Gefahren für die menschliche Gesundheit durch Krankheitserreger nicht entstehen. Einrichtungen zur Beseitigung des in Satz 1 genannten Abwassers unterliegen der infektionshygienischen Überwachung durch die zuständige Behörde.

2. Die Landesregierungen werden ermächtigt, bezüglich des Abwassers durch Rechtsverordnung entsprechende Gebote und Verbote zur Verhütung übertragbarer Krankheiten zu erlassen. Die Landesregierungen können die Ermächtigung durch Rechtsverordnung auf andere Stellen übertragen. Das Grundrecht der Unverletzlichkeit der Wohnung (\$13 Absatz 1 Grundgesetz) kann insoweit eingeschränkt werden.

Abwässer aus Krankenhäusern und anderen medizinischen Einrichtungen werden der kommunalen Abwasserreinigung zugeführt und damit nicht anders behandelt als Abwässer aus privaten Haushalten und öffentlichen Einrichtungen. Eine rechtliche Verpflichtung zur Vorbehandlung von
Abwasser, das in Krankenhäusern anfällt, besteht derzeit nicht. In der Verordnung über Anforderungen an das Einleiten von Abwasser in Gewässer (Abwasserverordnung) werden Krankenhausabwässer unter „häusliches Abwasser" eingruppiert.

Die Deutsche Vereinigung für Wasserwirtschaft, Abwasser und Abfall (DWA) hat bereits 2010 das Merkblatt DWA-M 775 Abwasser aus Krankenhäusern und anderen medizinischen Einrichtungen u.a. aufgrund des vermehrten Auftretens multiresistenter Mikroorganismen neu konzipiert. Allerdings gibt es bis heute keine einheitliche wissenschaftliche Auffassung, aus der sich eindeutige Handlungsempfehlungen für oder gegen eine dezentrale Vorbehandlung von Krankenhausabwässern ableiten lassen.

\section{Abwasserassoziierte Infektionsrisiken}

In den letzten Jahren haben sich wissenschaftliche Publikationen zum Vorkommen von antibiotikaresistenten Erregern einschließlich von Reserve-Antibiotika wie Carbapenemen und Colistin in Abwässern gehäuft [6-21].

In der Fördermaßnahme des Bundesministeriums für Bildung und Forschung (BMBF) „RiSKWa: Risikomanagement von neuen Schadstoffen und Krankheitserregern im Wasserkreislauf " und in dem nachfolgend vom BMBF geförderten Verbundvorhaben (HyReKA-Projekt) [22] wurden u.a. Untersuchungen zum Vorkommen von antibiotikaresistenten Bakterien, Resistenzgenen und Antibiotikarückständen durchgeführt. Dabei konnte u. a. festgestellt werden, dass in Klinikabwässern die höchsten Dichten an Antibiotika-Resistenzgenen nachgewiesen wurden [23]. Als eine der Kernbotschaften aus dem RiSKWa-Projekt wurde ermittelt, dass Abwassersysteme u. U. wichtige Punktquellen für die Belastung der aquatischen Umwelt in Bezug auf Krankheitserreger sowie klinisch relevante Antibiotikaresistenzen sein können.

In dem HyReKA-Projekt konnte gezeigt werden, dass Abwassersysteme aus Krankenhäusern in weit höherem $\mathrm{Maß}$ mit carbapenemresistenten Enterobacterales (CRE) und Carbapenemasen belastet sind im Vergleich zu Abwasser- systemen ohne Klinikeinfluss [24-26]. Kritische Kontrollpunkte im Verlauf des Abwassersystems eines Krankenhauses sind von Toiletten, Waschbecken und Duschabläufen beginnend, über Abwassersammler der Klinik und zentralem Abwassersammler des gesamten Klinikums, Zulauf und Ablauf der Kläranlage bis in das Gewässer in $\bullet$ Abb. 1 beispielhaft dargestellt.

Nachfolgend wird nur der mit (I) gekennzeichnete Abschnitt des Abwassersystems im Verantwortungsbereich der medizinischen Einrichtung behandelt.

\subsection{Infektionserreger}

$\mathrm{Zu}$ den wichtigsten in abwasserführenden Systemen nachgewiesenen fakultativ-pathogenen Infektionserregern zählen u. a.:

- Pseudomonas aeruginosa;

- Acinetobacter spp.;

- Enterobacterales wie E. coli, Klebsiella pneumoniae und Klebsiella oxytoca, Enterobacter spp., Citrobacter spp., Serratia spp., Raoultella spp., Morganella morganii, Leclercia spp;

- Clostridioides difficile;

- Enterokokken.

Besonders kritisch ist, wenn es sich dabei um antibiotikaresistente Erreger mit Resistenz gegen Carbapeneme und Colistin sowie dem Vorkommen einer erworbenen Carbapenemase handelt. Daneben sind auch Noroviren, Adenoviren und Enteroviren in die Risikobetrachtung einzubeziehen.

\section{2 Übertragung}

Die Übertragung von Infektionserregern (einschließlich antibiotikaresistenter Erreger) durch das Zurückspritzen beim Wasserablauf in Waschbecken oder Duschen bzw. bei Betätigung der Toilettenspülung (insbesondere bei Tiefspülern) ist beschrieben [27-31].

Das Design von Wasch- und Duschbecken mit direkter Abflussöffnung in der Mitte der Becken und nachfolgender Aerosol- bzw. Tröpfchenbildung beim Wasserablauf $[32,33]$ ist zukünftig verstärkt unter dem Gesichtspunkt der Rückübertragung von antibiotikaresistenten Erregern und anderen fakultativ pathogenen 


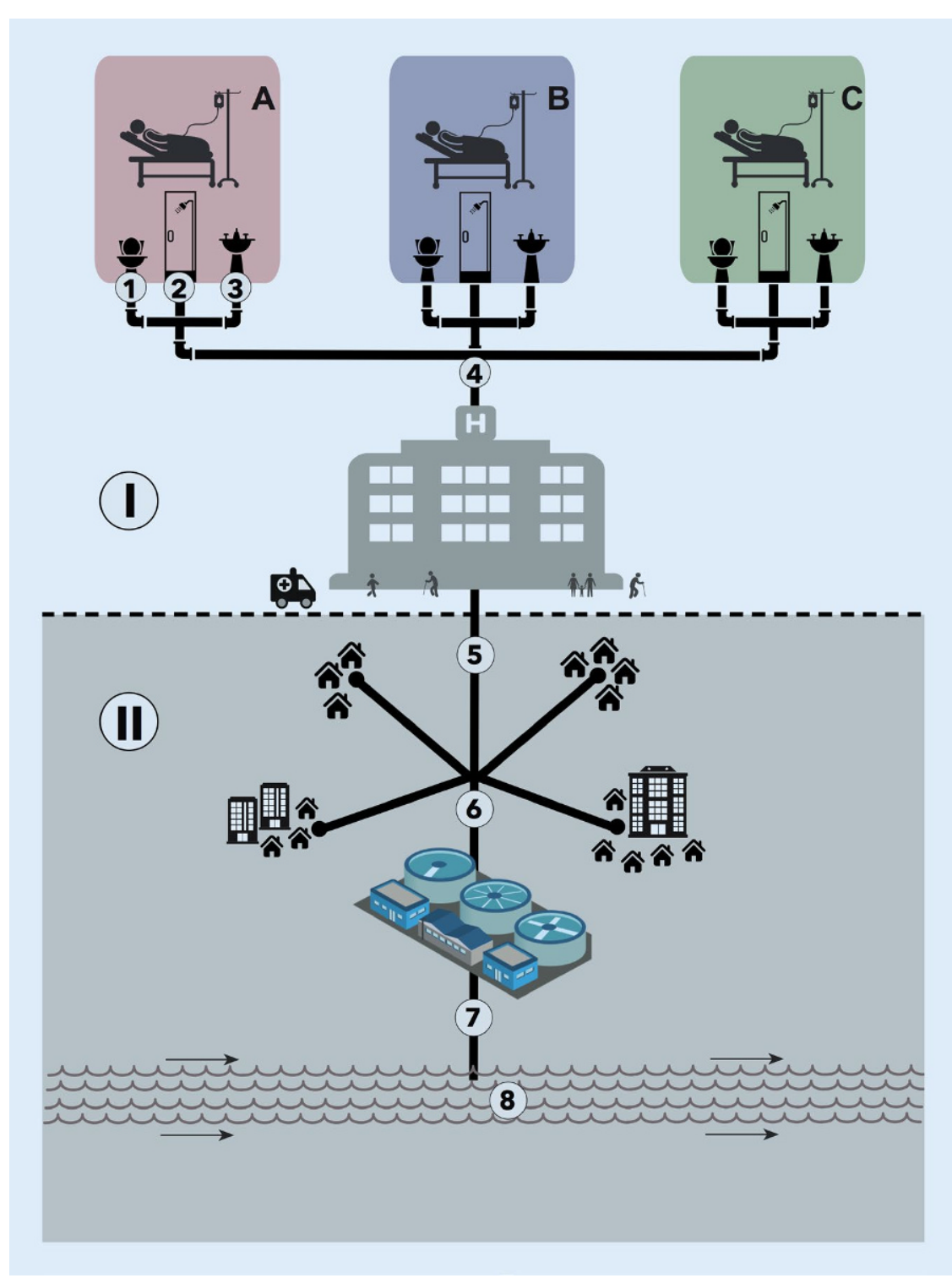

Abb. 1 A Abwasserpfade und Probenahmestellen von (1) Toiletten, (2) Duschabläufen und (3) Waschbeckenabläufen, (4) Abwassersammler der entsprechenden Klinik, (5) Zentraler Abwassersammler des gesamten Klinikums, (6) Zulauf der Kläranlage aus der Kommune, (7) Ablauf der Kläranlage, (8) in Flüssen flussabwärts der Kläranlage, im Rahmen des HyReKa-Projektes. Der Verantwortungsbereich der medizinischen Einrichtung bezieht sich auf den mit (I) gekennzeichneten Abschnitt des Abwassersystems, der Verantwortungsbereich des kommunalen Abwasserentsorgers bezieht sich auf den mit (II), grau unterlegten Abschnitt des Abwassersystems. (@ Dr. M. Parcina, Zentrum für Infektiologie und Infektionsprävention, Universitätsklinikum Bonn)

Mikroorganismen auf Patienten und medizinisches Personal als begünstigender Risikofaktor zu berücksichtigen. Es müssen konstruktive Lösungen gefunden werden, die die Aerosolbildung unterbinden.

In gleicher Weise können um das Waschbecken gelagerte Utensilien (etwa Waschlappen, Handtücher im Patientenbereich, Einmalhandschuhe, Desinfektionslösungen) sowie Utensilien bei der Zubereitung von Lösungen durch medi- zeigte sich, dass eine mögliche Kontaminationsquelle die abendliche Reinigung der Krankenhausküche mittels Hochdruckreinigungsgeräten ist (s. Ziffer 6.4.7 „Abwasserführendes System in Krankenhausküchen"). Aus den abwasserführenden Systemen und der Abwasseröffnung in den Küchen können sich durch das Absprühen mit hohem Druck Aerosole bilden, die nachfolgend Tüllen, Kochtöpfe und andere Küchenutensilien kontaminieren können. Dadurch ließe sich die Kontamination von Lebensmitteln (Pudding und Salat) erklären, über die sich die Übertragung auf Patienten in den unterschiedlichen Bereichen der betroffenen Klinik ereignete. Andere Kontaminationsmöglichkeiten ergaben sich durch eine Verbindung der Dunstabzugshaube mit dem Abwassersystem (vorgesehen für regelmäßige Spülungen der Abzugsbleche), die eine hochriskante bauliche Verbindung des Abwassersystems mit dem Kücheninventar darstellte. Nach Abstellen der Reinigung mit Hochdruckreinigungsgeräten und Umstellen auf Wischdesinfektionsverfahren für die Reinigung von Fußböden, der Demontage des Abflusssystems an der Abzugshaube und zeitweiliger Einstellung der Kaltspeisenversorgung, kam es zu vollständigem Sistieren des Ausbruchs [34]. Im Rahmen der Untersuchung dieses Ausbruchs konnten im zentralen Abwassersammelsystem des Krankenhauses unterschiedliche Carbapenemasen nachgewiesen werden.

Faulde et al. weisen auf einen wichtigen Indikator für unzureichende Sanitärhygiene hin, wobei es sich um die Infestation durch Clogmia albipunctata (Schmetterlingsmücken) handelt [35]. Diese haben die Eigenheit, sich in nicht ausreichend gespülten Abflüssen von Toiletten und Duschen anzusiedeln und ihre stark behaarten Körper und Flügel mit gramnegativen fakultativ pathogenen Erregern $\mathrm{zu}$ kontaminieren. Bei weitergehenden Untersuchungen konnten Faulde et al. bis zu 40 Genera auf Schmetterlingsmücken nachweisen, wozu Acinetobacter baumannii, Aeromonas hydrophila, Alcaligenes faecalis, Bacillus cereus, Escherichia coli, Klebsiella pneumoniae ssp. pneumoniae, Pseudomonas aeruginosa, P. fluorescens und Stenotrophomonas maltophilia zählten [36]. Inwieweit es hierdurch zur 
Übertragung nosokomialer Infektionen kommt, ist bislang ungeklärt. Eine Infestation mit Clogmia albipunctata in patientennahen Sanitärbereichen ist ein potenzieller Risikofaktor, daher unerwünscht und unter Kontrolle zu bringen [37-39].

\subsection{Wirksamkeit \\ abwasserassoziierter \\ Hygienemaßnahmen}

\subsubsection{Nosokomiale Infektionsausbrüche}

Als nosokomiale Infektionen in Assoziation mit dem abwasserführenden System sind sowohl sporadische Infektionen als auch lang dauernde Ausbrüche beschrieben. Ein Teil der beschriebenen Ausbrüche konnte durch entsprechende Maßnahmen am abwasserführenden System unter Kontrolle gebracht werden. Zwei umfassende Reviews haben den Kenntnisstand zu nosokomialen Ausbrüchen im Zusammenhang mit dem wasser- und abwasserführenden System zusammengefasst $[40,41]$.

Das systematische Review von Kizny Gordon et al. [40] umfasst nur den für Probennahmen erreichbaren Bereich bis einschließlich zum Siphon und konzentriert sich ausschließlich auf Berichte über carbapenemresistente Mikroorganismen als Indikator für die nosokomiale Bedeutung des abwasserführenden Systems. In diesem Review wurden 32 Berichte über carbapenemresistente Organismen mit Reservoir im abwasserführenden System ausgewertet, die nachfolgend näher beschrieben werden. In der Mehrzahl handelt es sich um Ausbrüche auf Intensivstationen und hämato-onkologischen Stationen, die hauptsächlich kritisch kranke und immunsupprimierte Personen betrafen. Hinsichtlich der Übertragung von Carbapenemasen wird festgestellt, dass eine Übertragung der Carbapenemresistenz innerhalb der wasser- und abwasserführenden Systeme stattfindet.

Im Ergebnis der Umgebungsuntersuchungen erwiesen sich Waschbecken mit ihren Abläufen und Armaturen (Wasserhähne) am häufigsten als kontaminiert. Dabei war Pseudomonas aeruginosa der vorherrschende Erreger. Die häufigsten nachgewiesenen Carbapenemasen waren Imipenemase (IMP), Klebsiella pneumo- niae Carbapenemase (KPC) und Verona-integron-encoded Metallo-Beta-Laktamase (VIM). Über eine Kontrolle des Ausbruchs berichten $72 \%$ der Studien, in mehr als einem Drittel durch Eliminierung der MRE in wasser- und abwasserführenden Systemen. Demnach ist die Kombination von Interventionsmaßnahmen einschließlich allgemeiner Infektionskontrollmaßnahmen und chemischer Desinfektion erfolgversprechend. Die Länge des Krankenhausaufenthalts vor Kolonisation/Infektion wurde nur in zehn Studien dokumentiert und variierte zwischen 1-134 Tagen.

In sechs der ausgewerteten Studien wurde die Kontamination der Hände, in zwei des Pharynx und in einer Studie rektale und nasale Kolonisation untersucht [40]. In einem Ausbruch mit imipenemresistenten Acinetobacter (IRAB) konnte die IRAB auf den Händen von zwei Pflegern nachgewiesen werden, die zuvor nicht in die Händehygiene eingewiesen worden waren. Nach Kohortierung und Händehygienetraining konnte der Ausbruch erfolgreich unter Kontrolle gebracht werden [42].

In allen Studien zeigten sich Hinweise für eine Kreuzübertragung zwischen $\mathrm{Pa}$ tienten und deren Umfeld hauptsächlich aufgrund epidemiologischer Zusammenhänge und/oder gleicher Antibiotikaresistenz bzw. molekularer Typisierung [40].

In neun Studien standen folgende Faktoren in ursächlichem Zusammenhang mit dem Ausbruch [40]:

- ungünstiges Design des Waschbeckens z. B. mit hoher Tröpfchenemission, Wasserhahn direkt über Waschbeckenablauf, Vorhandensein von Überlauf;

- Verwendung von Waschbecken für die Entsorgung von Schmutzwasser;

- Aufbewahrung von Patientenmaterialien (Zahnbürsten, Handschuhe) im unmittelbaren Umfeld von Waschbecken und Ausgussbecken;

- Verwendung einer einzigen Bürste, um Waschbecken ohne Zwischendesinfektion zu reinigen;

- Verstopfung von Abwasserleitungen mit Rückstau in das Waschbecken;

- Leckagen der Abwasserleitung;

- fehlerhafte Reinigung von Duschabläufen.
Die Exposition von Patienten und Personal resultierte aus direktem und indirektem Wasserkontakt oder durch Tröpfchen, die bei wasser- bzw. abwasserbezogenen Aktivitäten entstehen.

Interventionsmaßnahmen zur Eliminierung der carbapenemresistenten Mikroorganismen wurden in 27 Studien berichtet [40]; davon umfassten 25, zum Teil als Bündel:

- Maßnahmen zur Dekontamination des wasser- und abwasserführenden Systems einschließlich chemischer Desinfektion (mit Alkohol, Chlor, Aldehyden, Biguaniden, Natriumhypochlorit, Essigsäure, Wasserstoffperoxid, Silbernitrat, heißem Wasser und gespanntem Wasserdampf);

- Verwendung sterilen Wassers zum Waschen von Hochrisikopatienten;

- Nutzen von Waschbecken ausschließlich für die Händehygiene;

- Austausch von kontaminierten Ausstattungsmaterialien, Wasserarmaturen, Waschbecken oder DrainageSystemen.

Über die Einbeziehung allgemeiner Infektionskontrollmaßnahmen einschließlich Kontaktisolierung, strikter Händehygiene, aktiver Surveillance, Verbesserung der Reinigungs- und Desinfektionsprogramme, Audit und Schulungsprogramme berichten 22 Studien. Von 25 Studien mit spezifischen Umweltinterventionen konnte in 22 Studien hierdurch das klinische Ausbruchsgeschehen unter Kontrolle gebracht werden, wobei in etwas mehr als zwei Drittel der Fälle auch die Erreger aus den Umweltreservoiren erfolgreich eliminiert werden konnten. Erfolgreiche Interventionsmaßnahmen schlossen hierbei die Desinfektion von wasserführenden Systemen ein, inklusive der Reinigung von Waschbecken und Wasserhähnen, täglicher Reinigung der Waschbeckenoberflächen mit 0,1\% Natriumhypochlorit, wöchentlicher Reinigung der Waschbecken und des Installationssystems mit Essigsäure/heißem Wasser, Überführung aller Patienten in entsprechende Isolierungseinheiten und Raumdesinfektion mit Wasserstoffperoxid und des Ersatzes von Waschbecken oder Drainagesystemen. Daraus ergeben sich baulich-funktionelle Möglichkeiten zur Prävention und 
Kontrolle der von wasser- und abwasserführenden Systemen ausgehenden Infektionsrisiken. Dies konnte jedoch nur in circa einem Drittel der berichteten Fälle erreicht werden. Bei Ausbrüchen durch $P$. aeruginosa führte der Ersatz der Wasserarmaturen in circa zwei Drittel der Fälle zu einer Beendigung des Ausbruchs. Der Austausch von Waschbecken mit zentralem Abfluss und Siphon war als Teil von Bündelmaßnahmen erfolgreich bei der Beendigung von Ausbrüchen durch Enterobakterien (Klebsiella oxytoca) [43, 44], versagte aber bei $P$. aeruginosa. Dies wird verständlich, wenn es aus kontaminierten Wasserarmaturen mit $P$. aeruginosa immer wieder zur Rekontamination der Siphons kommt.

Andere, in einigen Fällen erfolgreiche Strategien beinhalteten:

- die Veränderung des Designs von Abflüssen und Waschbecken;

- die Verwendung von steril filtriertem Wasser für die Pflege von Hochrisikopatienten und

- die Vermeidung des Aufbewahrens von Patientenmaterialien um das Waschbecken.

In einer weiteren zusammenfassenden Publikation über nosokomiale Ausbrüche und deren Assoziation zu wasser- und abwasserführenden Systemen von Parkes und Hota [41] werden die Ergebnisse hinsichtlich der bislang übersehenen Bedeutung dieser Reservoire und die Empfehlungen von Kizny Gordon et al. bestätigt. Auch hier wird die Notwendigkeit von baulich-funktionellen Kriterien (insbesondere bei Neubauten), der Konstruktion von Waschbecken als auch von Desinfektionsmaßnahmen herausgestellt.

Ausbrüche, die mit Abwässern assoziiert sind, können polymikrobiell u.a. durch Plasmidtransfer verursacht sein. Bei einem Polyspezies Metallo-Beta-Laktamase German Imipenemase (GIM)1-Ausbruch auf einer chirurgischen Intensivstation und einer internistischen Station konnten 43 Beta-LaktamaseGene $\left(b l a_{\mathrm{GIM}-1}\right)$-tragende Bakterien als ursächlich nachgewiesen werden. Darunter befanden sich überwiegend Enterobacter cloacae und P. aeruginosa, die auch aus klinischem Material nachgewiesen wurden. Als Hauptreservoire wurden 30 Wasch- becken und ein Haarwaschbecken identifiziert. GIM konnte in 12 unterschiedlichen Spezies und Colistinresistenz in zwei E. cloacae-Isolaten detektiert werden. Die Umgebung mit einem zu vermutenden Multispezies-Biofilm stellt nach Ansicht der Autoren eine wichtige biologische Nische für multiresistente Bakterien und Resistenzgene dar [45].

In Zusammenhang mit diesem mehr als 10 Jahre dauernden Ausbruch wurden Waschbecken als Infektionsreservoir identifiziert [45]. Die Untersuchung des Biofilms zeigte, dass neben E. cloacae und Serratia marcescens sowie Citrobacter freundii und C. amalonaticus eine Vielzahl von Nonfermentern nachgewiesen werden konnte, die zur typischen aquatischen Mikroflora gehören. Nicht-fermentierende Bakterien haben zwar meist eine geringere klinische Bedeutung, können jedoch als genetisches Reservoir für Antibiotikaresistenzen dienen, was von erheblicher krankenhaushygienischer Bedeutung ist. Die Autoren schlussfolgern, dass Biofilme:

- in typischen Lokalisationen wie Waschbeckenabläufen mit einer heterogenen bakteriellen Population wichtige biologische Nischen für MRE und Antibiotika-resistenzgene darstellen;

- als Schmelztiegel für den horizontalen Gentransfer und die Ausbreitung in neue Spezies angesehen werden müssen und

- als Ausgangspunkt für Ausbrüche dienen können [45].

\subsubsection{Vorkommen gramnegativer Infektionserreger}

Mittlerweile liegen Studien über die Erfolge abwasserassoziierter Kontrollmaßnahmen auf die endemische Infektionsrate mit gramnegativen Infektionserregern vor, die von Parkes et al. mit dem Schwerpunkt waschbeckenbezogener Kontrollmaßnahmen zusammenfassend dargestellt sind [41]. Auf ausgewählte Studien wird nachfolgend eingegangen.

Hopman et al. [46] untersuchten die Auswirkung der Entfernung von Waschbecken aus den Patientenzimmern einer Intensivstation und der Einführung einer wasserfreien Patientenpflege auf die Rate nosokomial erworbener gramnegativer Bakterien. $\mathrm{Zu}$ der wasserfreien Patientenpflege gehörte, statt des Hän- dewaschens nach sichtbarer Verunreinigung, die Hände mit desinfektionsmittelgetränkten Tüchern und nachfolgender Händedesinfektion zu behandeln. Für die Zubereitung von Medikamentenlösungen und Getränken wurde ausschließlich abgepacktes Wasser aus Mineralwasserflaschen verwendet. Die Haarwäsche erfolgte mit spülfreien Shampoo-Kappen und die Körperwäsche durch Einmalwaschhandschuhe, die mit kommerziell erhältlichem Flaschenwasser angefeuchtet waren. Die Zahnpflege erfolgte ebenfalls mit abgepacktem Mineralwasser aus Mineralwasserflaschen und die Rasur entweder mit Elektrorasierern oder erwärmtem Wasser aus abgepacktem Mineralwasser. Diese Maßnahmen sowie die Entfernung von Waschbecken aus den Patientenzimmern der Intensivpflegestation führten zur signifikanten Reduktion der Patientenkolonisation mit gramnegativen Bakterien speziell bei Patienten mit längerem Aufenthalt.

In einer ähnlichen Studie wurde eine signifikante Reduktion der Inzidenz von multiresistenten gramnegativen Bakterien (MRGN) nach Einführung einer Wassersicherheitsstrategie mit Entfernung der Waschbecken aus Patientenräumen nachgewiesen [47].

Kossow et al. führten eine Untersuchung zur Kontrolle von multiresistenten $P$. aeruginosa auf einer hämato-onkologischen Station nach Einführung einer Bündelstrategie für das Sanitär- und Wasserversorgungssystem durch [48]. Zu den Maßnahmen zählten geänderte Abwasserabflüsse in Duschen, selbstdesinfizierende Siphons unterhalb der Patientenwaschbecken und die Einführung spülrandloser Toiletten. Im Vergleich zur Präinterventionsphase wurden wenige Kolonisationen und keine neue Infektion mit multiresistenten $P$. aeruginosa nachgewiesen. In dieser Studie war das neu eingeführte Design der Duschwasserabflüsse charakterisiert durch eine schwere, nicht rostende Stahlplatte über der Duschabflussöffnung, die so konstruiert war, dass sie leicht zu reinigen und desinfizieren ist. Die Verschlusskappe kann leicht entfernt und dekontaminiert werden (siehe auch $\bullet$ Tab. 2 im Anhang).

Eine weitere Studie untersuchte die Auswirkung des Einbaus von selbstdesin- 
fizierenden Siphons in Waschbecken auf das Vorkommen endemischer multiresistenter P. aeruginosa-Stämme [49]. Es handelte sich um eine kontrollierte klinische zweiarmige Studie, die in zwei Phasen in zwei Abteilungen einer Intensivstation durchgeführt wurde. Zunächst wurden nur in einer Abteilung alle Siphons durch Geräte ersetzt, die Hitze (mindestens $85^{\circ} \mathrm{C}$ ) und elektromechanische Vibration zum darin enthaltenen Wasser applizierten. Als Kontrolle dienten neue Siphons aus PVC, die in der anderen Abteilung eingebaut wurden. In der zweiten Phase wurden die desinfizierenden Geräte auch in der zweiten Abteilung eingebaut. Nach der Intervention waren sowohl die Kontaminationsrate von Waschbecken als auch die Kolonisationsrate von Patienten mit $P$. aeruginosa signifikant gesunken. Die Ergebnisse weisen darauf hin, dass eine thermische Desinfektion plus elektromechanische Vibration unterstützend bei Ausbruchskontrollen als Teil eines Maßnahmenbündels eingesetzt werden kann.

\subsection{Einzelne Bereiche des abwasserführenden Systems}

\subsubsection{Waschbecken}

Bereits 1967 berichtet Kohn [50] über seine langjährigen Erfahrungen zu Pseudomonaden-Infektionen in Krankenhäusern. Seine Schlussfolgerungen sind im Kontext mit abwasserführenden Systemen aus heutiger Sicht wegweisend. Insbesondere Siphons und Waschbecken seien zweifellos Reservoire und können Quellen für Kreuzübertragungen sein. In zahlreichen Fällen von Kolonisation oder Infektion konnten die Stämme bereits im Siphon von Waschbecken nachgewiesen werden, bevor der Patient in das Zimmer verlegt wurde.

Die Bedeutung der Tröpfchen- bzw. Aerosolbildung während des Händewaschens aus kontaminierten Abflüssen und die Übertragung auf Hände des Krankenhauspersonals konnte durch Döring et al. bestätigt werden [32]. So wurde in einer vierwöchigen prospektiven epidemiologischen Studie $P$. aeruginosa sowohl aus Abflüssen von Waschbecken, Duschen, Toiletten und Badewannen als auch von Personal und Patienten einer Infektionsstation eines deutschen Kinderkranken- hauses isoliert. Dabei waren $81 \%$ aller Abflüsse kontaminiert. Am Arbeitsbeginn waren alle Personalhände in Bezug auf $P$. aeruginosa negativ, während des Dienstes trugen $42,5 \%$ des Personals verschiedene $P$. aeruginosa-Stämme auf ihren Händen. Die mikrobiologische Auswertung ließ auf einen Übertragungsweg vom Abfluss der Waschbecken auf die Hände schließen. Als Interventionsmaßnahme war das Erhitzen der Waschbeckenabflüsse auf $70^{\circ} \mathrm{C}$ mit einer Vorrichtung bei der bakteriellen Wachstumshemmung in den Abflüssen sowie bei der Vermeidung $P$. aeruginosa-haltiger Aerosole erfolgreich. Das Händewaschen an derart ausgestatteten Waschbecken ergab $P$. aeruginosa-negative Handkulturen nach dem Waschen.

Als Kontaminationsquelle konnte ebenso der Überlauf des Waschbeckens im Zusammenhang mit einem schweren Serratia liquefaciens-Sepsisfall von Engelhart et al. belegt werden [29]. Auf die Bedeutung von Biofilmen in Waschbeckenabflüssen und die unzureichende Ausstattung von Waschbecken sowie Gestaltung von Patientenzimmern bei der Ausbreitung eines Pseudomonaden-Ausbruchs wiesen Hota et al. 2009 hin [30]. Die Quelle des Ausbruchs konnte auf Abflüsse in Handwaschbecken zurückgeführt werden, worin Biofilme und die entsprechenden vermehrungsfähigen Erreger nachgewiesen wurden. Die Untersuchung zeigte, dass sich bei Benutzen des Waschbeckens für die Händewaschung der Inhalt aus den Abflüssen mindestens einen Meter um das Waschbecken herum ausbreitete. Verschiedene Versuche, die Abläufe zu desinfizieren, schlugen fehl und der Ausbruch konnte nur durch Renovierung der Waschbecken zur Vermeidung von Spritzern in das Umfeld unter Kontrolle gebracht werden.

Salm et al. [51] berichten über Waschbecken als möglichen Übertragungsweg im Zusammenhang mit einem multiresistenten $P$. aeruginosa-Ausbruch auf einer interdisziplinären Intensivstation. Im Rahmen des Ausbruchmanagements konnte der Ausbruchsstamm in fünf von 16 Patientenräumen nachgewiesen werden. Der Aufenthalt in einem Patientenzimmer mit einem kontaminierten Waschbecken war unabhängig assoziiert mit einem erhöhten Risiko für Kolonisa- tion oder Infektion durch den Ausbruchsstamm. Daraufhin wurden die Siphons der kontaminierten Waschbecken ausgetauscht und alle Arbeitsvorgänge eliminiert, die unter Einbeziehung von Waschbecken in Patientenräumen durchgeführt wurden, um Rückspritzwasser um die Waschbecken zu vermeiden. Die Rate an Infektionen konnte auf diese Weise drastisch reduziert werden.

In einem weiteren langdauernden Ausbruch (im Zusammenhang mit einem Polyspecies Metallo-Beta-Laktamase GIM-1 durch E. cloacae und P. aeruginosa) konnten Wendel et al. bei Umgebungsuntersuchungen in Waschbecken $b l a_{\mathrm{GIM}-1}$-tragende Bakterien (v.a. Nonfermenter als auch Enterobacteriaceae) in verschiedene Waschbecken einer chirurgischen Intensivstation nachweisen $[45,52]$. Durch eingeschränkte Nutzung der Waschbecken (keine Entnahme von Wasser für patientennahe Tätigkeiten, kein Aufsetzen von Gegenständen) konnte der Ausbruch unter Kontrolle gebracht werden.

Bezüglich Nutzungsverhalten, welches Waschbecken als potentielles Reservoir für antibiotikaresistente Bakterien bei nosokomialen Ausbrüchen begünstigt, führten Grabowski et al. eine Beobachtungsstudie durch [53]. Hierzu wurden in über 4800 Videos die Aktivitäten des medizinischen Personals an Handwaschbecken in einer Intensivstation ausgewertet. Lediglich in $17 \%$ der Fälle fanden Maßnahmen der Händehygiene statt (davon war das eigentliche Händewaschen in nur $4 \%$ der Fälle beobachtet). Vielmehr erfolgten regelmäßig verschiedene nicht Händehygiene spezifische Aktivitäten, von medizinischen Pflegemaßnahmen (Spritzenzubereitung, Ausleeren von intravenösen Lösungen, etc.) über Verarbeitung der patienteneigenen Speisen oder Getränke bis hin zu Reinigungsmaßnahmen (Reinigen des Waschbeckens etc.).

Experimentelle Untersuchungen an einer Galerie von Waschbecken ergaben, dass von einem künstlich mit E. coli kontaminierten Waschbecken über die gleiche Abwasserleitung weitere Waschbecken kontaminiert werden können [54]. In der Klinik konnten Hopman et al. gleiche Carbapenemase-produzierende $P$. aeruginosa in den Duschabflüssen unterschiedlicher Patientenzimmer nachweisen, was eben- 
falls ein Hinweis dafür sein kann, dass über die Abwasserleitung eine Verbindung zu unterschiedlichen Patientenzimmern auch über das Abwassersystem bestehen kann [55].

Des Weiteren untersuchten AranegaBou et al. in einem Labormodellsystem die Ausbreitung carbapenemresistenter Enterobacterales aus Waschbecken in Abhängigkeit von der Position des Abflusses und der Abflussrate [33]. Bei artifizieller Kontamination von Waschbeckenabflüssen mit CRE wurden signifikant weniger Bakterien von solchen Waschbecken abgegeben, aus denen ein rascher $\mathrm{Ab}$ fluss gewährleistet wurde und/oder von Waschbecken, deren Abfluss sich rückseitig im Waschbecken befand. Bei natürlicher Kontamination der Waschbeckenabflüsse mit CRE fand sich ebenfalls eine signifikante Wechselwirkung zwischen Waschbeckenabfluss und Positionierung der Waschbeckenabflüsse. Bei langsamem Ablauf wurden von rückseitig positionierten Abflüssen im Waschbecken 30-fach weniger Bakterien abgegeben als von Waschbecken, deren Abfluss unterhalb des Wasserzulaufes positioniert war. Wenn ein rascher Abfluss gewährleistet war, gaben Waschbecken mit rückseitig positionierten Abflüssen relativ weniger carbapenemresistente Enterobacterales $\mathrm{ab}$, obwohl in diesen Fällen die Differenz statistisch nicht signifikant war. Kontaminierte Tröpfchen breiteten sich wie auch in den Untersuchungen von Hopman et al. circa einen Meter um das Waschbecken aus. Von Bedeutung ist daher der zusätzliche Aspekt des raschen Ablaufs und der Positionierung des Abflusses im Waschbecken (siehe auch $\bullet$ Tab. 2 im Anhang).

\subsubsection{Toiletten}

Die Bedeutung von Toiletten als Reservoir für 4MRGN P. aeruginosa wurde von Engelhart et al. beschrieben [27]. Im Rahmen von Screeninguntersuchungen fiel auf einer hämato-onkologischen Schwerpunktstation ein Anstieg der Inzidenzdichte von 4MRGN $P$. aeruginosa-Besiedlungen auf, denen zunächst mit klassischen Hygienemaßnahmen zu begegnen versucht wurde (Basishygiene- und Isolationsmaßnahmen, Schulungen, erhöhte Hygienefachkräftepräsenz, Quellensuche mittels Umgebungsuntersuchung etc.).
Erst der Nachweis von Kontaminationen mit multiresistenten $P$. aeruginosa unter den Spülrändern mehrerer Toiletten und der komplette Austausch dieser Toilettenschüsseln durch spülrandlose Modelle führte zu einem raschen nachhaltigen Absinken der Inzidenzdichte. Die Autoren schlussfolgern, dass Toiletten mit Spülrändern zu einer dauerhaften Besiedlungsquelle für $P$. aeruginosa werden können. Daher führte im vorliegenden Fall nur der Ersatz durch spülrandlose Modelle zur Lösung der Problematik. Spülrandlose Toiletten zählten ebenfalls in den Untersuchungen von Kossow et al. [48] (im Rahmen einer Bündelstrategie für das Sanitär- und Wasserversorgungssystem) zur Kontrolle von multiresistenten $P$. aeruginosa auf einer hämato-onkologischen Station.

Das bei der Toilettenspülung entstehende Aerosol stellt eine andere mögliche Kontaminationsquelle dar, denn Erreger wie Legionella spp. können bei Kontamination des Kaltwassersystems über das Aerosol aufgenommen werden. Der Bezug zwischen Legionellen und Toilettenspülungen wurde erstmals 2007 von der Weltgesundheitsorganisation (WHO) im Zusammenhang mit Brauchwasserlösungen diskutiert [56]. Allerdings ist die Expositionsdosis aus Toilettenaerosol deutlich geringer als aus Dusche oder Waschbecken. Das Schließen des Toilettendeckels vor dem Spülen reduziert die Kontaminationsgefahr zusätzlich um mehr als das 10-fache [57].

\subsubsection{Steckbecken}

Bei Steckbeckenspülgeräten werden thermische, chemothermische und chemische Geräte unterschieden, wobei nur thermische Steckbeckenspülgeräte den aktuellen normativen Anforderungen entsprechen. Die relevante Norm für diesen Bereich ist die DIN EN ISO 15883-3. In ihr wird als Mindestwert für die thermische Desinfektion von Steckbecken ein $\mathrm{A}_{0}$-Wert von 60 aufgeführt. Es wird dort keine wissenschaftliche Begründung für diesen Wert aufgeführt. Hingegen wird in der allgemeinen Norm DIN EN ISO 15883-1 zum $\mathrm{A}_{0}$-Wert von 60 aufgeführt: „Es wird betont, dass hierfür eine geringe mikrobiologische Belastung vor der Desinfektion und die Abwesenheit hitzeresistenter Or- ganismen, die ernste Krankheiten beim Menschen hervorrufen können, vorliegen müssen.“. Bei anderen unkritischen Medizinprodukten wird von verschiedenen Fachgesellschaften im Allgemeinen ein Mindest- $\mathrm{A}_{0}$-Wert von 600 für die $\mathrm{Ab}$ tötung bzw. Inaktivierung von Bakterien inklusive Mykobakterien, Pilze und thermolabile Viren gefordert [58-60]. Es wurde auch beschrieben, dass ein $\mathrm{A}_{0}$-Wert von 60 nicht ausreichend ist, um die erforderliche thermische Desinfektion zu erreichen [61-64].

\subsubsection{Duschen und Duschabläufe}

Die Bedeutung von Duschabläufen als Erregerreservoir ist unklar. Im Rahmen von Ausbrüchen wurde in verschiedenen Fällen neben der Kontamination von Waschbecken auch eine Kontamination der Duschabläufe festgestellt und die Abläufe in die Maßnahmen mit einbezogen.

So waren neben Waschbecken auch Duschabläufe in einem von Longtin et al. beschriebenen Ausbruch kontaminiert [31]. Zwischen 2006 und 2008 wurden auf einer pädiatrischen Intensivstation eines Schweizer Universitätsklinikums 37 Fälle von $P$. aeruginosa-Infektionen (einschließlich drei tödlich verlaufender Septikämien) sowie -Kolonisationen festgestellt. Ein eng verwandter Stamm wurde bei Kindern, die vor und nach einer Herzoperation in einem Nichtregierungsorganisations-Aufnahmezentrum untergebracht waren, nachgewiesen. Von vier zusätzlichen Bewohnern (nach Hospitalaufnahme) und aus einem Abfluss in dem Aufnahmezentrum konnte P. aeruginosa isoliert werden. Bei Bewohnern wurde $65 \%$ der Gesamtanzahl von $P$. aeruginosa-Kolonisationen oder -Infektionen festgestellt. Die Untersuchung des Aufnahmezentrums zeigte eine ausgedehnte Kontamination des Abwassersystems bei 10 von 14 Waschbecken und Duschabläufen (70 \%) und eine hohe Prävalenz (38\%) von $P$. aeruginosa-Trägern unter den Kindern. Die Autoren schlussfolgern, dass die wahrscheinliche Ursache für diesen Ausbruch die Kontamination des Abwassersystems des Aufnahmezentrums war, mit konsekutiver nosokomialer Übertragung nach Aufnahme in die Klinik. Hierdurch konnte auch die Bedeutung der Überprüfung externer 
Quellen außerhalb von Krankenhäusern belegt werden.

Ferner wurde die Bedeutung von Abflüssen als Reservoir für ESBL-produzierende E. cloacae von Chapuis et al. gezeigt [28]. In deren Untersuchung wurde ein Ausbruch mit Beta-Laktamase-produzierenden E. cloacae mit hoher Toleranz gegenüber quaternären Ammoniumverbindungen (QAV) auf einer hämatoonkologischen Station mit dem kontaminierten Sanitärbereich assoziiert. Es wurden 17 ESBL-produzierende (ESBL, Beta-Laktamasen mit breitem Wirkungsspektrum) E. cloacae in den Patientenwaschbeckenabläufen, Duschabläufen und Abläufen von Ausgussbecken identifiziert. Die minimalen Hemmkonzentrationen $(\mathrm{MHK})$ der Isolate gegen die quaternäre Ammoniumverbindung, die für die Desinfektion verwendet wurden, waren sowohl bei den klinischen als auch bei den Umgebungsisolaten außerordentlich hoch. Unmittelbar nach Implementierung eines Desinfektionsprogramms mit Chlorbleichlauge konnte ein substantieller Abfall in den Fallzahlen verzeichnet werden. Folglich betont die Untersuchung die Notwendigkeit, dass Abflüsse der korrekten mechanischen Reinigung zugänglich sein müssen und entsprechende Desinfektionsmittel mit nachgewiesener Wirksamkeit gegenüber nosokomialen Krankheitserregern (z. B. auf Chlorbasis anstelle von QAV) verwendet werden müssen.

Außerdem beschreiben Kossow et al. das Design von sterilisierbaren Duschabläufen auf einer hämato-onkologischen Station als Teil eines Bündels von Sanitärmaßnahmen, in deren Folge es zu einer deutlichen Abnahme von P. aeruginosa-Kolonisationen und -Infektionen kam [48].

Hopman et al. beschreiben eine letal verlaufende krankenhauserworbene Infektion durch carbapenemase-bildende $P$. aeruginosa, die die Autoren auf Luftübertragung beim Duschen zurückführten. Darüber hinaus wurde die $P$. aerugino$s a$-Weiterverbreitung über die Abwasserleitung zu sieben weiteren Patientenzimmern nachgewiesen [55]. Durch tägliche Desinfektion mit einer $0,1 \%$-Chlorlösung gelang eine Reduktion, die nach Erhöhung des Reinigungsintervalls auf eine Woche wieder zu einem Wiederauftreten von $P$. aeruginosa in den Abwasserproben führte. Aus diesem Grunde wurde die Reinigungsfrequenz wieder auf einmal täglich erhöht.

\subsubsection{Abwasserführendes Leitungssystem im Krankenhaus}

Die Bedeutung des abwasserführenden Systems zeigt sich in Ausbrüchen, bei denen nach anfänglichem Erfolg von Maßnahmen an den peripheren Sanitärobjekten erneute Nachweise auf eine Rekontamination aus den tieferen Bestandteilen des Abwassersystems schließen lassen. Im Folgenden werden wesentliche Untersuchungen hierzu beschrieben.

Vergara-Lopez et al. [43] beschreiben das abwasserführende System als verstecktes Reservoir von Metallo-BetaLaktamase-produzierenden $K$. oxytoca mit Verursachung eines klonalen Ausbruchs auf der Intensivstation eines spanischen Krankenhauses zwischen 2009 und 2011, der in vier Wellen ablief. Insgesamt waren 42 Patienten betroffen. Das Screening der Mitarbeiter der Intensivstation war wiederholt negativ. Die Umgebungsuntersuchungen einschließlich trockener Flächen waren ebenso negativ. Die ersten Basismaßnahmen (aktive Surveillance, Kontaktmaßnahmen und verstärkte Oberflächenreinigung) und zusätzliche Kontrollmaßnahmen (Kohortierung des medizinischen Personals und Verbesserung des Patienten-PflegepersonalVerhältnisses) wurden eingeführt. Diese Maßnahmen führten vorübergehend zu einer Reduktion, aber nicht zu einer Eradikation des endemischen multiresistenten K. oxytoca-Stamms, der zwei Wochen nach Entlassung der kolonisierten Patienten in Welle 2 und 3 wieder auftrat. Erst die Verbesserung der horizontalen Abwasserführung führte schließlich zu einem Stopp des Ausbruchs. Die Autoren schlussfolgern, dass Kreuzübertragungen in Feuchtbereichen (hauptsächlich Abflüsse, Siphons und horizontale abwasserführende Systeme) Ursache für die fehlgeschlagene Kontrolle von Ausbrüchen sein können. Derartige Reservoire sollten nach Empfehlung der $\mathrm{Au}$ toren mit einbezogen werden, wenn Umgebungs- oder Oberflächenuntersuchungen im Kontext mit Ausbruchuntersuchungen negativ sind.
Auf das Abwasserleitungssystem als mögliche Quelle für einen Acinetobacter baumannii-Ausbruch auf einer Intensivstation geht La Forgia ein [65]. Zwischen 2004 und 2005 kam es bei 16 Patienten auf einer gemischt chirurgischen und internistischen Intensivstation zu Infektionen mit einem multiresistenten A. baumanniiStamm. Dabei hatten 11 der 16 infizierten Patienten einen klonal identischen multiresistenten Stamm. Das Reservoir des $A$. baumannii-Ausbruchstammes konnte in einem Siphon in einem der Patientenräume festgestellt werden, der wahrscheinlich die Kontamination des gesamten horizontalen Abwassersystems repräsentierte. Die Behandlung des Abwassersystems mit Chlorbleichlauge führte zur erfolgreichen Dekontamination des Abwassersystems und zur Kontrolle der Infektionen.

Von Breathnach et al. [66] wurden 2012 multiresistente PseudomonadenAusbrüche in zwei Krankenhäusern mit dem kontaminierten Krankenhausabwassersystem in Verbindung gebracht; jeder beinhaltete einen distinkten $\mathrm{Ge}$ notyp von multiresistenten Pseudomonaden. Der eine Ausbruch betraf das gesamte Krankenhaus mit insgesamt 85 Patienten; der andere konzentrierte sich lediglich auf 4 Fälle in einer spezialisierten internistischen Intensivstation. Die ausgedehnten Umgebungsuntersuchungen wiesen in beiden Ausbrüchen auf das abwasserführende System hin. Die Inspektion und die Zustandsbeschreibung zeigten zahlreiche Faktoren, die zu einer Kontamination von klinischen Bereichen führen können, wie:

- fehlerhaft konstruierte Waschbecken, bei denen der Wasserstrahl direkt auf die Abflussöffnung gerichtet ist und es zur Aerosolentstehung kommt;

- ungünstiges Design von Toiletten und Duschbecken;

- Aufbewahrung von reinen Materialien in der Nähe von Abflüssen und

- häufige Verstopfung und Leckagen von abwasserführenden Systemen.

Die Verstopfung war häufig auf Toilettenpapier, Patientenfeuchttücher und fehlerhafte Anwendung von Steckbeckenspülern zurückzuführen.

Die Kontrollmaßnahmen beinhalteten: 
- den Ersatz von Waschbecken und Toiletten durch leichter zu reinigende Modelle, die zu weniger Spritzern führten;

- die Schulung der Mitarbeiter, um Verstopfungen und unsachgerechte Aufbewahrung zu vermeiden;

- die Anpassung der Reinigungsmaßnahmen und

- die Vermeidung von Überflutungen durch Reduzierung der Flussraten der Duschen.

Hierdurch wurde eine signifikante Reduktion von Infektionen und Kolonisationen festgestellt.

\subsubsection{Entleerung von Dialysebeuteln}

Ross et al. [67] beschrieben Ablaufanschlüsse zur Entleerung von Dialysatbeuteln als eine mögliche Infektionsquelle für carbapenemresistente KPC-produzierende Enterobacterales (Klebsiella oxytoca, Citrobacter freundii, Klebsiella pneumoniae). Durch Optimierung der Entsorgung einschließlich strikter Händedesinfektion nach Kontakt mit den Abläufen und einer Desinfektion derselben mittels Chlorlösung konnte der Ausbruch unter Kontrolle gebracht werden.

\subsubsection{Abwasserführendes System in Krankenhausküchen}

Im Zeitraum zwischen Oktober 2013 und September 2014 kam es zu einem Ausbruch mit unterschiedlichen KPC-2-produzierenden Enterobakterien bei $132 \mathrm{~Pa}$ tienten eines deutschen Krankenhauses [34]. Bemerkenswert war, dass bei nahezu einem Drittel der Patienten mehr als eine KPC-2-produzierende Spezies nachgewiesen werden konnte [34, 68]. Die Analyse des Ausbreitungsgeschehens deutete darauf hin, dass es sich um eine abteilungsübergreifende Quelle im Krankenhaus handelte. Daher wurde die Küche als möglicher Infektionsherd in Betracht gezogen. Dort konnte aus einer Abwasseröffnung Citrobacter freundii nachgewiesen werden, der zu den KPC-2-tragenden Enterobacterales zählte. Im weiteren Verlauf erwies sich das Abwassersystem der Küche mit diesem Erreger, der zuvor bereits in Toiletten, Waschbecken, Siphons und Duschabläufen unterschiedlichster
Stationen nachgewiesen worden war, als kontaminiert, worüber auch die in der Küche hergestellten Kaltspeisen mit diesen Enterobacterales kontaminiert wurden. Die Kontamination des Abwassersystems der Küche, das nicht mit dem Abwassersystem der Stationen im Zusammenhang stand, erfolgte mit hoher Wahrscheinlichkeit durch eine Reinigungsspirale, die sowohl in der Küche als auch in den Patientenzimmern bei verstopften Abflüssen eingesetzt wurde. An der Reinigungsspirale konnten KPC-2-tragenden Enterobakterien nachgewiesen werden. Dieser Ausbruch war in vielerlei Hinsicht ungewöhnlich:

- es handelte sich um einen Multispezies-Ausbruch mit KPC-2-produzierenden Enterobakterien;

- es betraf eine ungewöhnlich hohe Zahl an Patienten;

- die Ausbreitung erfolgte sehr wahrscheinlich über eine zentral streuende Quelle (Nahrungsmittel);

- eine Ausbreitung eines KPC-2-Plasmids über sekundär kontaminierte Lebensmittel aus einem Umwelt-/Abwasserreservoir wurde bislang nicht beschrieben;

- es konnte gezeigt werden, dass ein Resistenzgen auf einem mobilen Element auf eine Vielzahl von Spezies verteilt werden kann;

- die Kontamination der Lebensmittel und von Gerätschaften in der Küche erfolgte mutmaßlich u. a. über Spritzer bei der abendlichen Hochdruckreinigung der Küche. Dabei konnten während des Reinigungsvorganges in der Raumluft u. a. Enterobakterien und Acinetobacter spp. nachgewiesen werden.

\section{7. Ökologie, Reservoire und mit abwasserführenden Systemen assoziierte Übertragung}

Die in abwasserführenden Systemen hauptsächlich vorkommenden gramnegativen Bakterien weisen zahlreiche Besonderheiten auf, die ihre Persistenz und Resistenz in aquatischen Biotopen abwasserführender Systeme begünstigen:

- gramnegative Bakterien sind zum großen Teil adaptiert an aquatische
Biotope und teilweise selbst Teil der aquatischen Mikroflora [69];

- Enterobakterien, Pseudomonaden und Acinetobacter sind in der Lage, Biofilme zu bilden. Biofime ermöglichen ihnen nicht nur Schutz und Persistenzvermögen, sondern auch Austausch von Informationen sowie eine erhöhte Antibiotikaresistenz und Toleranz gegenüber Desinfektionsmittelwirkstoffen [70];

- auch Clostridioides difficile lässt sich in abwasserführenden Systemen nachweisen und weist eine spezifische Toleranz gegenüber Desinfektionsmitteln auf [71, 72];

- Enterobakterien weisen häufig eine hohe MHK gegenüber quaternären Ammoniumverbindungen auf [28, 73];

- gramnegative Erreger in Assoziation mit Abwässern zeichnen sich durch hohe Anspruchslosigkeit aus [74-77] und sind in der Lage, über einen weiten Temperaturbereich vermehrungsfähig zu bleiben [75, 78];

- etliche Enterobacterales und andere nicht-fermentierende Bakterien sind in der Lage, in sog. „viable but nonculturable"(VBNC) Status überzugehen. Hierdurch entgehen sie dem kulturbasierten Nachweis und lassen die entsprechenden Infektionsreservoire nicht erkennen [79].

\subsection{Desinfektionswirkstoffe zur Elimination von Biofilmen}

Chapuis et al. [28] konnten im Zusammenhang mit abwasserassoziierten Ausbrüchen mit Enterobakterien zeigen, dass insbesondere E. cloacae, aber auch andere Enterobakterien offensichtlich intrinsisch eine hohe Toleranz gegenüber quaternären Ammoniumverbindungen aufweisen. Dies gilt insbesondere für die Anwesenheit von Effluxpumpengenen, die bereits bei E. coli und K. pneumoniae beschrieben wurden. Bei Vorkommen von Enterobakterien im Biofilm in Waschbeckenabflüssen können bei nur kurzzeitiger Einwirkung von Desinfektionsmitteln auf der Basis von QAV nur subinhibitorische Konzentrationen einwirken, sodass $\mathrm{Mi}$ kroorganismen trotz häufiger Desinfektion überleben können. Darüber hinaus 
besteht die Möglichkeit, dass es zur Entwicklung einer erhöhten Toleranz gegenüber QAV kommt. Nach Umstellung des Desinfektionsmittels von QAV auf Chlorbleichlauge war die Anzahl der kolonisierten bzw. infizierten Patienten mit antibiotikaresistenten Erregern deutlich rückläufig. Ein Überblick über die Adaptation, Abbau und Ökologie von Mikroorganismen und QAV wird von Tezel et al. gegeben [80].

Aldehyde konservieren Biofilme an Oberflächen und sind ohne vorherige mechanische Reinigung nicht zur Desinfektion biofilmtragender Oberflächen in abwasserführenden Systemen geeignet [81]. In verschiedenen Untersuchungen zur Kontrolle von abwasserassoziierten nosokomialen Infektionen konnten durch Desinfektion mit Chlorprodukten wie auch mit Perverbindungen gute Erfolge erzielt werden $[55,65]$.

Einschränkend ist zu bemerken, dass keine standardisierten Methoden zur Überprüfung der Desinfektionswirkung in abwasserführenden Systemen etabliert sind, sodass über geeignete Konzentrationen und Einwirkzeiten noch erheblicher Forschungsbedarf besteht.

\subsection{Abwasserführende Systeme als Reservoir für die Ausbreitung von Antibiotikaresistenzen}

Das im Siphon stehende Abwasser bildet ein ideales Biotop zur Vermehrung fakultativ pathogener gramnegativer Mikroorganismen, die sich darüber hinaus in Biofilmen an Oberflächen von Abflusssieben, Abflussdeckeln usw. bei Zimmertemperatur halten. Zudem werden über die Abflüsse von Waschbecken, Toiletten, Duschabläufen und Entsorgungsbecken antibiotikaenthaltende Körperflüssigkeiten und Ausscheidungen in das Abwasser abgegeben (z. B. Sekrete, Exkrete, Patientenflüssigkeiten wie synoviale, perikardiale, pleurale und peritoneale Flüssigkeiten, Hämofiltrationslösungen, Dialysat etc.).

Rückstände dieser Antibiotika lassen sich teilweise in hoher Konzentration in verschiedenen Klinikabwässern nachweisen. Nach Untersuchungen von Voigt et al. [26] konnten in Toiletten, Waschbeckenabflüssen und Duschabläufen Antibiotikarückstände von Carbapenemen, Cephalo- sporinen, Fluorchinolonen, Lincosamiden, Makroliden, Nitroimidazolen, Penicillinen, Glycopeptiden, Sulfonamiden (inklusive Trimethoprim) und Tetrazyklinen in Konzentrationen von bis zu 79 mg/l nachgewiesen werden. Hierbei handelt es sich um Konzentrationen, die z. T. weit über den von Bengtsson-Palme und Larsson genannten PNEC-Konzentrationen (Predicted No Effect Concentrations) für die Resistenzselektion liegen [82], sodass Selektionsprozesse zur Ausbildung von Antibiotikaresistenzen bei nosokomialen Infektionserregern im unmittelbaren Umfeld des Patienten induziert werden beziehungsweise bereits bestehende Antibiotikaresistenzen persistieren können.

Im Rahmen des HyReKA-Verbundvorhabens wurden unter Berücksichtigung der o.a. Erkenntnisse Sanitärbereiche (Waschbecken, Abflüsse, Toiletten und Duschabflüsse) in drei unterschiedlichen Kliniken (Psychosomatik, Hämato-Onkologie und Neurologische Rehabilitation) auf das Vorkommen von antibiotikaresistenten Erregern und Antibiotikarückständen untersucht $[25,26]$. Antibiotikaresistente Bakterien konnten aus den Siphons aller Kliniken isoliert werden, wobei es einen Zusammenhang zwischen der Art, dem Einsatz von Antibiotika und der Frequenz des Nachweises multiresistenter Erreger gab. Insgesamt zeigte sich, dass die Belastung an Antibiotikarückständen und antibiotikaresistenten Bakterien in den Sanitärbereichen stark von den Antibiotikaverbrauchszahlen der untersuchten Kliniken abhängig ist $[25,26]$. Die Mehrzahl der multiresistenten Erreger sowie besonders häufige Nachweise an Antibiotikarückständen (oberhalb der PNEC für die Resistenzselektion) wurden in den Abflüssen von Duschbecken nachgewiesen. Nach 24-stündiger Stagnation ohne Benutzung kam es zu einem Wiederanstieg der Antibiotikarückstandskonzentrationen, die durch Spülen reduziert wurden. Gleichartig verhielten sich Nachweise der multiresistenten Erreger, indem nach mechanischer Reinigung ohne Erreichen der in der Tiefe befindlichen Biofilme zunächst keine antibiotikaresistenten Bakterien festgestellt wurden. Dennoch wurde nach 24-stündiger Stagnation eine retrograde Kontamination mit multiresistenten Bakterien im Biofilm nachgewiesen.
Um den Biofilm zu reduzieren, sollten nach Auffassung der Autoren insbesondere in Bereichen mit immunsupprimierten Patienten die Siphons täglich desinfizierend gespült werden. Darüber hinaus sei die regelmäßige Entfernung von hoch kontaminierten Biofilmen insbesondere in Duschabläufen von Bedeutung. Durch ein optimiertes Design von Duschabläufen, die leicht zu reinigen oder zu ersetzen sind, könnte die Persistenz in Abwasser und eine Rückübertragung zum Patienten verringert werden $[25,26]$.

\section{Empfehlungen}

Die in den letzten Jahren weltweit festgestellte zunehmende Carbapenemresistenz und das verbesserte Verfahren $\mathrm{zu}$ deren Nachweis in der Umwelt hat die Aufmerksamkeit auf das Abwassersystem als Reservoir und Quelle für gramnegative Erreger gelenkt. Dabei ist eine Reihe von Fragen über die genauen Übertragungswege und deren Unterbrechung ungeklärt und es besteht Forschungsbedarf. Dennoch lässt sich bereits jetzt feststellen, dass Interventionen begründbar sind und diese zukünftig durch epidemiologische Daten weiter abgesichert werden sollen.

In Abhängigkeit vom Einsatz von Antibiotika einerseits und der Infektionsgefährdung der Patienten andererseits ist es sinnvoll, die Anforderungen an abwasserführende Systeme und den Umgang mit diesen zu differenzieren. In medizinischen Einrichtungen kann orientiert an der KRINKO-Empfehlung Anforderungen an die Hygiene bei der Reinigung und Desinfektion von Flächen zwischen Bereichen ohne, mit möglichem und mit besonderem Risiko unterschieden werden. Es muss jedoch berücksichtigt werden, dass die Abwassersysteme in medizinischen Einrichtungen untereinander verbunden sind und sich bestimmte Risiken damit ggf. nicht auf bestimmte Bereiche begrenzen lassen.

$\mathrm{Zu}$ den Bereichen ohne Infektionsrisiko zählen Bereiche ohne Nutzung durch Patienten wie Verwaltung, Hörsäle, Unterrichtsräume, Toiletten für Angehörige, Speisesäle für medizinisches Personal.

Bereiche mit möglichem Infektionsrisiko umfassen alle Bereiche, in denen Patienten mit invasiven Systemen (In- 
fusionssysteme, Harnwegskatheter), mit Verletzung von Haut und Schleimhaut, oder unter oraler wie parenteraler Antibiotikatherapie bestimmungsgemäß behandelt werden, soweit sie nicht unter die nächsthöhere Risikogruppe fallen.

$\mathrm{Zu}$ den Bereichen mit besonderem Infektionsrisiko zählen alle Bereiche, in denen bestimmungsgemäß Patienten mit erhöhtem Infektionsrisiko oder Patienten, für die ein erhöhter Antibiotikaeinsatz erforderlich ist, gepflegt werden. Dazu gehören z.B. Bereiche für immunsupprimierte/-defiziente Patienten mit hohem bzw. sehr hohem Infektionsrisiko (Risikogruppe 2 und 3) [3], polytraumatisierte Patienten, Schwerstbrandverletzte, Intensivtherapiepatienten einschließlich neonatologischen Patienten, Weaning-Patienten, Patienten in der neurologischen Frührehabilitation.

In den folgenden Empfehlungen wird auf grundsätzliche Anforderungen der Hygiene an abwasserführende Systeme in medizinischen Einrichtungen eingegangen. Darüber hinaus werden in $\bullet$ Tab. 2 im Anhang Beispiele für Maßnahmen in den verschiedenen Regelungs- und Risikobereichen gegeben, die hilfreich für die Umsetzung der Hygieneanforderungen sein können.

\subsection{Aufklärung, Information, Schulung und Organisation}

\section{Die Kommission empfiehlt:}

- im Rahmen der Schulungen zur Basishygiene auf die Bedeutung des abwasserführenden Systems als Reservoir für nosokomiale Infektionserreger (einschließlich antibiotikaresistente Erreger), Resistenzgene und Antibiotikarückstände sowie auf Präventions- und Kontrollmaßnahmen einzugehen (Kat. II);

- im Rahmen von Prozessbeobachtungen auch die Nutzung und Aufbereitung von Waschbecken durch das Personal zu berücksichtigen (Kat. II);

- Patienten über die hygienisch sichere Nutzung der Sanitäreinheiten zu informieren (ohne Kat.).

\subsection{Design von Waschbecken, sanitären Anlagen und Duschen}

Bei der Ausstattung von Waschbecken gelten die Empfehlungen der KRINKO zur Händehygiene in Einrichtungen des Gesundheitswesens [4]. Durch bauliche Vorkehrungen und geeignete Prozesse kann die Kontaminationsgefahr aus Waschbecken, Duschen und WCs reduziert werden.

\section{Die Kommission empfiehlt:}

- infektionspräventive Aspekte beim Design von Waschbecken, Duschen und WCs bei zukünftigen Umbauund Neubauplanungen zu prüfen (Kat. IV).

Beispiele hierzu gibt $\bullet$ Tab. 2 im Anhang.

\subsection{Desinfektion im Sanitärbereich}

Die Kommission empfiehlt:

- zur anlassbezogenen Sanierung von kontaminierten abwasserführenden Systemen bei Waschbecken, Abflussöffnungen, Duschbecken und Toiletten bevorzugt Desinfektionsmittelpräparate auf Peroxid- oder Chlorbasis z. B. nach Entlassung oder Verlegung von mit 4MRGN kolonisierten oder infizierten Patienten, zur Desinfektion zu verwenden (Kat. II).

Weiteres zur Desinfektion von Waschbecken, Duschen und WCs befindet sich in - Tab. 2 im Anhang.

\subsection{Ausgussbecken in unreinen Räumen}

Die Kommission empfiehlt:

- Regelungen zur Entsorgung kontaminierter Flüssigkeit und zu persönlichen Schutzmaßnahmen in den Hygieneplan aufzunehmen (Kat. II).

Beispielhafte Hinweise zum Design von Ausgussbecken befinden sich in $\bullet$ Tab. 2 im Anhang.

\subsection{Steckbeckenspüler}

Die Kommission empfiehlt:

- Regelungen zum $\mathrm{A}_{0}$-Wert und zur Nachreinigung bei sichtbarer Verunreinigung in den Hygieneplan aufzunehmen (ohne Kat.).

Weitere Hinweise zur Ausstattung mit Steckbeckenspülern und zu deren baulichfunktioneller Anordnung an strategisch sinnvollen Stellen und Zimmerzuordnung bei zukünftigen Umbau- und Neubauplanungen befinden sich in $\bullet$ Tab. 2 im Anhang.

\subsection{Abwasserführende Systeme}

\section{Die Kommission empfiehlt:}

- unter Bezug auf die allgemein anerkannten Regeln der Technik, Abwasserleitungen so zu betreiben, dass die Gefahr von Verstopfungen und Leckagen mit Rückstau in den Patientenbereich auf ein Mindestmaß reduziert wird (ohne Kat.)

\subsection{Maßnahmen bei Havariefällen von Abwasserleitungen}

\section{Die Kommission empfiehlt:}

- sicher zu stellen, dass das Hygienefachpersonal über Havariefälle in abwasserführenden Systemen mit Rückstau in den Patientenkontaktbereich informiert wird, um geeignete flankierende Schutzmaßnahmen festzulegen (Kat. II).

\subsection{Küchen}

Die Kommission empfiehlt:

- Reinigungs- und Desinfektionsverfahren im Bereich der Böden und Abflüsse und deren Überwachung unter Berücksichtigung der speziellen Anforderungen der Lebensmittelverarbeitung in den Hygieneplan aufzunehmen (Kat. II).

\subsection{Maßnahmen bei Ausbrüchen}

Die Kommission empfiehlt:

- bei gehäuftem Auftreten von abwasserassoziierten Krankheitserregern als mögliche Infektionsursache und im 


\section{Abkürzungen}

\begin{tabular}{|c|c|}
\hline bla & Beta-Laktamase-Gene \\
\hline$B M B F$ & $\begin{array}{l}\text { Bundesministerium für Bildung } \\
\text { und Forschung }\end{array}$ \\
\hline CRE & $\begin{array}{l}\text { Carbapenemresistente } \\
\text { Enterobacterales }\end{array}$ \\
\hline$D W A$ & $\begin{array}{l}\text { Deutsche Vereinigung für } \\
\text { Wasserwirtschaft, Abwasser } \\
\text { und Abfall }\end{array}$ \\
\hline $\begin{array}{l}\text { DWA-M } \\
775\end{array}$ & $\begin{array}{l}\text { Deutsche Vereinigung für } \\
\text { Wasserwirtschaft, Abwasser } \\
\text { und Abfall - Merkblatt } 775\end{array}$ \\
\hline$E S B L$ & $\begin{array}{l}\text { Beta-Laktamase mit breitem } \\
\text { Wirkungsspektrum }\end{array}$ \\
\hline GIM & German Imipenemase \\
\hline HyReKA & $\begin{array}{l}\text { Verbundprojekt des Bun- } \\
\text { desministeriums für Bildung } \\
\text { und Forschung (BMBF) zu } \\
\text { Antibiotikaresistenzen im } \\
\text { Wasserkreislauf; Biologische } \\
\text { bzw. hygienisch-medizinische } \\
\text { Relevanz und Kontrolle } \\
\text { antibiotikaresistenter Krank- } \\
\text { heitserreger in klinischen, } \\
\text { landwirtschaftlichen und } \\
\text { kommunalen Abwässern } \\
\text { und deren Bedeutung in } \\
\text { Rohwässern }\end{array}$ \\
\hline IMP & Imipenemase \\
\hline$I R A B$ & $\begin{array}{l}\text { Imipenemresistente Acineto- } \\
\text { bacter }\end{array}$ \\
\hline$K P C$ & $\begin{array}{l}\text { Klebsiella pneumoniae } \\
\text { Carbapenemase }\end{array}$ \\
\hline KRINKO & $\begin{array}{l}\text { Kommission für Krankenhaus- } \\
\text { hygiene und Infektionsprä- } \\
\text { vention }\end{array}$ \\
\hline MHK & Minimale Hemmkonzentration \\
\hline MRE & Multiresistente Erreger \\
\hline MRGN & $\begin{array}{l}\text { Multiresistente gramnegative } \\
\text { Bakterien }\end{array}$ \\
\hline NDM & $\begin{array}{l}\text { Neu-Delhi Metallo-Beta- } \\
\text { Laktamase }\end{array}$ \\
\hline PNEC & $\begin{array}{l}\text { Predicted No Effect Concent- } \\
\text { rations }\end{array}$ \\
\hline QAV & $\begin{array}{l}\text { Quaternäre Ammoniumverbin- } \\
\text { dungen }\end{array}$ \\
\hline RiSKWa & $\begin{array}{l}\text { Risikomanagement von neuen } \\
\text { Schadstoffen und Krankheitser- } \\
\text { regern im Wasserkreislauf }\end{array}$ \\
\hline VBNC & Viable but non-culturable \\
\hline VIM & $\begin{array}{l}\text { Verona-integron-encoded } \\
\text { Metallo-Beta-Laktamase }\end{array}$ \\
\hline WHO & Weltgesundheitsorganisation \\
\hline
\end{tabular}

Rahmen der Ausbruchsanalyse auch das abwasserführende System als potentielle Quelle mit in die Abklärung einzubeziehen (Kat. II).

Interessenkonflikt. Diese Empfehlung wurde ehrenamtlich und ohne Einflussnahme kommerzieller Interessengruppen im Auftrag der Kommission für Krankenhaushygiene und Infektionsprävention erarbeitet von Prof. Dr. Martin Exner (Leiter der Arbeitsgruppe und Koordinator des vom BMBF geförderten Verbundprojektes HyReKA), Prof. Dr. Steffen Engelhart, Prof. Dr. Axel Kramer, Dr. Dr. Ricarda Schmithausen, Prof. Dr. Matthias Trautmann und Prof. Dr. Constanze Wendt. Vom Robert Koch-Institut war Dr. (Univ. Lissabon) Vanda Marujo beteiligt. Die Empfehlung wurde durch die Arbeitsgruppe vorbereitet und nach ausführlicher Diskussion in der Kommission abgestimmt.

\section{Literatur}

1. Roca I, Akova M, Baquero F et al (2015) The global threat of antimicrobial resistance: science for intervention. New Microbes New Infect 6:22-29

2. Kommission für Krankenhaushygiene und Infektionsprävention (KRINKO) (2004) Anforderungen an die Hygiene bei der Reinigung und Desinfektion von Flächen. Bundesgesundheitsbl 47(1):51-61

3. Kommission für Krankenhaushygiene und Infektionsprävention (KRINKO) (2010) Anforderungen an die Hygiene bei der medizinischen Versorgung von immunsupprimierten Patienten. Bundesgesundheitsbl 53(4):357-388

4. Kommission für Krankenhaushygiene und Infektionsprävention (KRINKO) (2016) Händehygiene in Einrichtungen des Gesundheitswesens. Bundesgesundheitsbl 59(9):1189-1220

5. Infektionsschutzgesetz vom 20. Juli 2000 (BGBI.I S. 1045), das zuletzt durch Artikel 46 des Gesetzes vom 12. Dezember 2019 (BGBI. I S. 2652) geändert worden ist.

6. Amador PP, Fernandes RM, Prudencio MC, Barreto MP, Duarte IM (2015) Antibiotic resistance in wastewater: occurrence and fate of Enterobacteriaceae producers of class $A$ and class $C$ beta-lactamases. J Environ Sci Health A Tox Hazard Subst Environ Eng 50(1):26-39

7. Amos GC, Hawkey PM, Gaze WH, Wellington EM (2014) Waste water effluent contributes to the dissemination of CTX-M-15 in the natural environment. J Antimicrob Chemother 69(7):1785-1791

8. Baquero F, Martinez JL, Canton R (2008) Antibiotics and antibiotic resistance in water environments. Curr Opin Biotechnol 19(3):260-265

9. Blaustein RA, Shelton DR, Van Kessel JA, Karns JS, Stocker MD, Pachepsky YA (2015) Irrigation waters and pipe-based biofilms as sources for antibioticresistant bacteria. Environ Monit Assess 188(1):56

10. Chagas TP, Seki LM, Cury JC et al (2011) Multiresistance, beta-lactamase-encoding genes and bacterial diversity in hospital wastewater in Rio de Janeiro, Brazil. J Appl Microbiol 111(3):572-581

11. Chen H, Zhang M (2013) Occurrence and removal of antibiotic resistance genes in municipal wastewater and rural domestic sewage treatment systems in eastern China. Environ Int 55:9-14
12. Cheng W, Chen H, Su C, Yan S (2013) Abundance and persistence of antibiotic resistance genes in livestock farms: a comprehensive investigation in eastern China. Environ Int 61:1-7

13. Czekalski N, Sigdel R, Birtel J, Matthews B, Burgmann H (2015) Does human activity impact the natural antibiotic resistance background? Abundance of antibiotic resistance genes in 21 Swiss lakes. Environ Int 81:45-55

14. da Costa PM, Loureiro L, Matos AJ (2013) Transfer of multidrug-resistant bacteria between intermingled ecological niches: the interface between humans, animals and the environment. Int J Environ Res Public Health 10(1):278-294

15. Fuentefria DB, Ferreira AE, Graf T, Corcao G (2008) Pseudomonas aeruginosa: spread of antimicrobial resistance in hospital effluent and surface water. Rev Soc Bras Med Trop 41(5):470-473

16. Galvin S, Boyle F, Hickey P, Vellinga A, Morris D, Cormican M (2010) Enumeration and characterization of antimicrobial-resistant Escherichia coli bacteria in effluent from municipal, hospital, and secondary treatment facility sources. Appl Environ Microbiol 76(14):4772-4779

17. Gao L, Hu J, Zhang X et al (2014) Dissemination of ESBL-producing Escherichia coli of chicken origin to the nearby river water. J Mol Microbiol Biotechnol 24(4):279-285

18. Girlich D, Poirel L, Szczepanowski R, Schluter A, Nordmann P (2011) Carbapenem-hydrolyzing GES-5-encoding gene on different plasmid types recovered from a bacterial community in a sewage treatment plant. Appl Environ Microbiol 78(4):1292-1295

19. Gundogdu A, Jennison AV, Smith HV, Stratton H, Katouli M (2013) Extended-spectrum beta-lactamase producing Escherichia coli in hospital wastewaters and sewage treatment plants in Queensland, Australia. Can J Microbiol 59(11):737-745

20. Kistemann T, Rind E, Rechenburg A et al (2008) A comparison of efficiencies of microbiological pollution removal in six sewage treatment plants with different treatment systems. Int J Hyg Environ Health 211(5-6):534-545

21. Koh TH, Ko K, Jureen R et al (2015) High counts of carbapenemase-producing Enterobacteriaceae in hospital sewage. Infect Control Hosp Epidemiol 36(5):619-621

22. Exner M, Schwartz T, Universitätsklinikum Bonn, Karlsruher Institut für Technologie (2019) HyReKA: Biologische bzw. hygienisch-medizinische Relevanz und Kontrolle Antibiotika-resistenter Krankheitserreger in klinischen, landwirtschaftlichen und kommunalen Abwässern und deren Bedeutung in Rohwässern. http://www.hyreka. net/.Zugegriffen: 13. Jan. 2020

23. Exner M, Schwartz TH (2015) RiSKWa-Statuspapier. Bewertungskonzepte der Mikrobiologie mit den Schwerpunkten neue krankheitserreger und Antibiotikaresistenzen. Ergebnisse des Querschnittsthemas „Bewertungskonzepte der Mikrobiologie“. DECHEMA e. V.: Frankfurt am Main. http://riskwa.de/Downloads/_/RISKWA_Statuspapier_Mikrobiologie_2015_10_30.pdf.ZZugegriffen: 13. Jan. 2020

24. Müller H, Sib E, Gajdiss M et al (2018) Dissemination of multi-resistant Gram-negative bacteria into German wastewater and surface waters. FEMS Microbiol Ecol 94(5):fiy57

25. Sib E, Voigt AM, Wilbring G et al (2019) Antibiotic resistant bacteria and resistance genes in biofilms 
in clinical wastewater networks. Int J Hyg Environ Health 222(4):655-662

26. Voigt AM, Faerber HA, Wilbring G et al (2019) The occurrence of antimicrobial substances in toilet, sink and shower drainpipes of clinical units: A neglected source of antibiotic residues. Int J Hyg Environ Health 222(3):455-467

27. Engelhart S, Wolf D, Abels S, Exner M (2014) Toiletten als Reservoir für 4-fach resistente $P$. aeruginosa. Hyg Med 39(Suppl):13

28. Chapuis A, Amoureux L, Bador J et al (2016) Outbreak of Extended-Spectrum Beta-Lactamase Producing Enterobacter cloacae with High MICs of Quaternary Ammonium Compounds in a Hematology Ward Associated with Contaminated Sinks. Front Microbiol 7:1070. https://doi.org/10.3389/ fmicb.2016.01070

29. Engelhart S, Saborowski F, Krakau M, ScherholzSchlosser G, Heyer I, Exner M (2003) Severe Serratia liquefaciens sepsis following vitamin $C$ infusion treatment by a naturopathic practitioner. J Clin Microbiol 41(8):3986-3988

30. Hota S, Hirji Z, Stockton K et al (2009) Outbreak of multidrug-resistant Pseudomonas aeruginosa colonization and infection secondary to imperfect intensive care unit room design. Infect Control Hosp Epidemiol 30(1):25-33

31. Longtin $Y$, Troillet $N$, Touveneau $S$ et al (2009) Pseudomonas aeruginosa outbreak in a pediatric intensive care unit linked to a humanitarian organization residential center. Pediatr Infect Dis J 29(3):233-237

32. Döring G, Ulrich M, Müller W et al (1991) Generation of Pseudomonas aeruginosa aerosols during handwashing from contaminated sink drains, transmission to hands of hospital personnel, and its prevention by use of a new heating device. Zentralbl Hyg Umweltmed 191(5-6):494-505

33. Aranega-Bou P, George RP, Verlander NQ et al (2019) Carbapenem-resistant Enterobacteriaceae dispersal from sinks is linked to drain position and drainage rates in a laboratory model system. J Hosp Infect 102(1):63-69

34. Carstens A, Kepper U, Exner M, Hauri A, Kaase M, Wendt C (2014) Plasmid-vermittelter MultispeziesAusbruch mit Carbapenem-resistenten Enterobacteriaceae. Epid Bull 47:455-459

35. Faulde M, Spiesberger M (2012) Hospital infestations by the moth fly, Clogmia albipunctata (Diptera: Psychodinae), in Germany. J Hosp Infect 81(2):134-136

36. Faulde M, Spiesberger M (2013) Role of the moth fly Clogmia albipunctata (Diptera: Psychodinae) as a mechanical vector of bacterial pathogens in German hospitals. J Hosp Infect 83(1):51-60

37. Spiesberger M, Faulde M (2013) Die Schmetterlingsmücke Clogmia albipunctata als neuer mechanischer Vektor: aktuelle Verbreitung in Deutschland und ihr infektiologisches Potenzial in deutschen Krankenhäusern. Hyg Med 38(6):228

38. Faulde M (2013) Clogmia albipunctata: Befallsmonitoring von Schmetterlingsmücken mit Lichtklebe- und Ölfallen. Prakt Schädlingsbekämpfer 65(9):10-11

39. Faulde M (2013) Die Schmetterlingsmücke Clogmia albipunctata (Williston, 1893): Ein neuer Gebäude- und Hygieneschädling aus Südeuropa. Prakt Schädlingsbekämpfer 65(1):14-19

40. Kizny Gordon AE, Mathers AJ, Cheong EYL et al (2017) The Hospital Water Environment as a Reservoir for Carbapenem-Resistant Organisms Causing Hospital-Acquired Infections-A
Systematic Review of the Literature. Clin Infect Dis 64(10):1435-1444

41. Parkes LO, Hota SS (2018) Sink-Related Outbreaks and Mitigation Strategies in Healthcare Facilities. Curr Infect Dis Rep 20(10):42

42. Wang CH, Li JF, Huang LY et al (2017) Outbreak of imipenem-resistant Acinetobacter baumannii in different wards at a regional hospital related to untrained bedside caregivers. Am J Infect Control 45(10):1086-1090

43. Vergara-Lopez S, Dominguez MC, Conejo MC, Pascual A, Rodriguez-Bano J (2013) Wastewater drainage system as an occult reservoir in a protracted clonal outbreak due to metallo-beta-lactamaseproducing Klebsiella oxytoca. Clin Microbiol Infect 19(11):E490-E498

44. Leitner E, Zarfel G, Luxner J et al (2014) Contaminated handwashing sinks as the source of a clonal outbreak of KPC-2-producing Klebsiella oxytoca on a hematology ward. Antimicrob Agents Chemother 59(1):714-716

45. Wendel AF, Kolbe-Busch S, Ressina S et al (2015) Detection and termination of an extended lowfrequency hospital outbreak of GIM-1-producing Pseudomonas aeruginosa ST111 in Germany. Am J Infect Control 43(6):635-639

46. Hopman J, Tostmann A, Wertheim H et al (2017) Reduced rate of intensive care unit acquired gram-negative bacilli after removal of sinks and introduction of 'water-free' patient care. Antimicrob Resist Infect Control 6:59

47. Shaw E, Gavalda L, Camara J et al (2018) Control of endemic multidrug-resistant Gram-negative bacteria after removal of sinks and implementing a new water-safe policy in an intensive care unit. J Hosp Infect 98(3):275-281

48. Kossow A, Kampmeier S, Willems S et al (2017) Control of Multidrug-Resistant Pseudomonas aeruginosa in Allogeneic Hematopoietic Stem Cell Transplant Recipients by a Novel Bundle Including Remodeling of Sanitary and Water Supply Systems. Clin Infect Dis 65(6):935-942

49. de Jonge $E$, de Boer MGJ, van Essen EHR, Dogterom-Ballering HCM, Veldkamp KE (2019) Effects of a disinfection device on colonization of sink drains and patients during a prolonged outbreak of multidrug-resistant Pseudomonas aeruginosa in an intensive care unit. J Hosp Infect 102(1):70-74

50. Kohn J (1967) Pseudomonas infection in hospital. Br Med J 4(5578):548

51. Salm F, Deja M, Gastmeier P et al (2016) Prolonged outbreak of clonal MDR Pseudomonas aeruginosa on an intensive care unit: contaminated sinks and contamination of ultra-filtrate bags as possible route of transmission? Antimicrob Resist Infect Control 5:53

52. Wendel AF, Ressina S, Kolbe-Busch S, Pfeffer K, MacKenzie CR (2016) Species Diversity of Environmental GIM-1-Producing Bacteria Collected during a Long-Term Outbreak. Appl Environ Microbiol 82(12):3605-3610

53. Grabowski M, Lobo JM, Gunnell B et al (2018) Characterizations of handwashing sink activities in a single hospital medical intensive care unit. J Hosp Infect 100(3):e115-e122

54. Kotay S, Chai W, Guilford W, Barry K, Mathers AJ (2017) Spread from the Sink to the Patient: In Situ Study Using Green Fluorescent Protein (GFP)Expressing Escherichia coli To Model Bacterial Dispersion from Hand-Washing Sink-Trap Reservoirs. Appl Environ Microbiol 83(8):e3327-16

55. Hopman J, Meijer C, Kenters N et al (2019) Risk Assessment After a Severe Hospital-Acquired
Infection Associated With Carbapenemase-Producing Pseudomonas aeruginosa. Jama Netw Open 2(2):e187665

56. World Health Organization (WHO) (2007) Legionella and the prevention of legionellosis. WHO Press: Genf, Schweiz. https://apps.who.int/iris/ handle/10665/43233. Zugegriffen: 13. Jan. 2020

57. Best EL, Sandoe JA, Wilcox MH (2012) Potential for aerosolization of Clostridium difficile after flushing toilets: the role of toilet lids in reducing environmental contamination risk. J Hosp Infect 80(1):1-5

58. Popp W, Martiny H, Zastrow KD (2012) Aufbereitung von Medizinprodukten - Was ist eigentlich der A0-Wert? https://www.krankenhaushygiene.de/informationen/hygiene-tipp/hygienetipp2012/399. Zugegriffen: 13. Jan. 2020

59. Amann B, Bertram M et al (2018) Keine Aufbereitung von Nierenschalen und Waschschüsseln in Steckbeckenspülern. Zentralsterilization 26(2):108-111

60. Deutsche Gesellschaft für Krankenhaushygiene (DGKH), Deutsche Gesellschaft für Sterilgutversorgung (DGSV), Arbeitskreis Instrumentenaufbereitung (AKI) (2017) Leitlinie von DGKH, DGSV und AKI für die Validierung und Routineüberwachung maschineller Reinigungs- und thermischer Desinfektionsprozesse für Medizinprodukte, 5. Aufl. mhp Verlag, Wiesbaden

61. Bradley CR, Fraise AP (1996) Heat and chemical resistance of enterococci. J Hosp Infect 34(3):191196

62. van der Velden LB, Nabuurs-Franssen $M$, Leeuwen Av, Isken M, Voss A (2013) 0066: Thermal disinfection of bedpans: European ISO 15883-3 guideline requirements are insufficient to ensure elimination of ARE and OXA-48 outbreak-strains. Antimicrob Resist Infect Control 2(Suppl 1):066

63. Diab-Elschahawi M, Furnkranz U, Blacky A, Bachhofner N, Koller W (2010) Re-evaluation of current $\mathrm{A} 0$ value recommendations for thermal disinfection of reusable human waste containers based on new experimental data. J Hosp Infect 75(1):62-65

64. Rosenberg U (2003) Thermische Desinfektion das A0-Konzept und der biologische Hintergrund. Zentralsterilization 11(2):115-120

65. La Forgia C, Franke J, Hacek DM, Thomson RB Jr., Robicsek A, Peterson LR (2009) Management of a multidrug-resistant Acinetobacter baumannii outbreak in an intensive care unit using novel environmental disinfection: A 38-month report. Am J Infect Control 38(4):259-263

66. Breathnach AS, Cubbon MD, Karunaharan RN, Pope CF, Planche TD (2012) Multidrug-resistant Pseudomonas aeruginosa outbreaks in two hospitals: association with contaminated hospital waste-water systems. J Hosp Infect 82(1):19-24

67. Ross B, Krull M, Rath P et al (2019) Dialysis drains as a possible source for carbapenem-resistant pathogens causing an ICU outbreak. Infection 47(2):233-238

68. Carstens A (2015) Ausbruch von KPC-2 produzierenden multiresistenten bakterien in einer Klinik in Südhessen - Ausbruchmangement und Rolle des öffentlichen Gesundheitsamtes Hessisches. Ärzteblatt 4:196-198

69. Wingender J, Flemming HC (2011) Biofilms in drinking water and their role as reservoir for pathogens. Int J Hyg Environ Health 214(6):417-423

70. Flemming HC, Wingender J, Szewzyk U, Steinberg P, Rice SA, Kjelleberg S (2016) Biofilms: an emergent form of bacterial life. Nat Rev Microbiol 14(9):563-575 
71. Ali S, Muzslay M, Wilson P (2015) A Novel Quantitative Sampling Technique for Detection and Monitoring of Clostridium difficile Contamination in the Clinical Environment. J Clin Microbiol 53(8):2570-2574

72. Shaughnessy MK, Bobr A, Kuskowski MA et al (2016) Environmental Contamination in Households of Patients with Recurrent Clostridium difficile Infection. Appl Environ Microbiol 82(9):2686-2692

73. Sidhu MS, Sorum H, Holck A (2002) Resistance to quaternary ammonium compounds in foodrelated bacteria. Microb Drug Resist 8(4):393-399

74. Flemming HC (2016) EPS-Then and Now. Microorganisms. https://doi.org/10.3390/microorganisms 4040041

75. Obeidat N, Jawdat F, Al-Bakri AG, Shehabi AA (2014) Major biologic characteristics of Acinetobacter baumannii isolates from hospital environmental and patients' respiratory tract sources. Am J Infect Control 42(4):401-404

76. Flemming HC (2011) The perfect slime. Colloids Surf B Biointerfaces 86(2):251-259

77. Dwidjosiswojo Z, Richard J, Moritz MM, Dopp E, Flemming HC, Wingender J (2011) Influence of copper ions on the viability and cytotoxicity of Pseudomonas aeruginosa under conditions relevant to drinking water environments. Int J Hyg Environ Health 214(6):485-492

78. Brown AD (1957) Some general properties of a psychrophilic pseudomonad: the effects of temperature on some of these properties and the utilization of glucose by this organism and Pscudomonas aeruginosa. J Gen Microbiol 17(3):640-648

79. Bloomfield S, Exner M, Flemming HC et al (2015) Lesser-known or hidden reservoirs of infection and implications for adequate prevention strategies: Where to look and what to look for. GMS Hyg Infect Control 10:Doc4

80. Tezel U, Pavlostathis SG (2015) Quaternary ammonium disinfectants: microbial adaptation, degradation and ecology. Curr Opin Biotechnol 33:296-304

81. Exner M, Tuschewitzki GJ, Scharnagel J (1987) Influence of biofilms by chemical disinfectants and mechanical cleaning. Zentralbl Bakteriol Mikrobiol Hyg B 183(5-6):549-563

82. Bengtsson-Palme J, Larsson DG (2016) Concentrations of antibiotics predicted to select for resistant bacteria: Proposed limits for environmental regulation. Environ Int 86:140-149 


\section{Informativer Anhang}

Tab. 2 Informative Beispiele für Präventionsmaßnahmen für abwasserführende Systeme in medizinischen Einrichtungen

\begin{tabular}{l} 
Regelungsbereich \\
\hline $\begin{array}{l}\text { Sensibilisierung, Information } \\
\text { und Schulung des Personals }\end{array}$
\end{tabular}
$\begin{array}{lll}\begin{array}{l}\text { Bereich ohne } \\ \text { Infektionsrisiko (I) }\end{array} & \begin{array}{l}\text { Bereich mit möglichem } \\ \text { Infektionsrisiko (II) }\end{array} & \text { Bereich mit besonderem Infektionsrisiko (III) }\end{array}$

Im Rahmen von Schulungen, z. B. zur Basishygiene, wird auf die Bedeutung des abwasserführenden Systems als Reservoir für nosokomiale Infektionserreger (einschließlich antibiotikaresistente Erreger), für Resistenzgene und Antibiotikarückstände sowie auf geeignete Präventions- und Kontrollmaßnahmen eingegangen. Besondere Risiken werden adressiert, z. B. Probleme der Verstopfung durch Abwerfen von Wischtüchern in die Toilette.

Patienten

Waschbecken

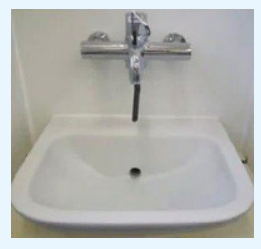

Toiletten

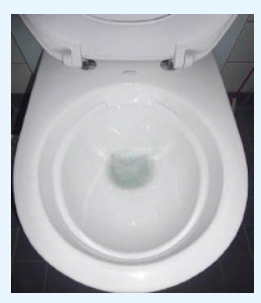

b
Keine besonderen Anforderungen
Keine besonderen Anforderungen

Sofern Patienten Sanitärbereiche nutzen, werden sie über die hygienegerechte Benutzung des Sanitärbereiches informiert (Waschbecken, spritzwassergeschützte Positionierung ihrer persönlichen Utensilien, Benutzung von Duschen und Toiletten) z. B. durch geeignete Informationsmaterialien.

Falls Arbeitsflächen für aseptische Tätigkeiten, z. B. Umgang mit Infusionslösungen, Parenteralia und Ernährungslösungen, an den Waschplatz angrenzen, sind diese durch Spritzschutz abzuschirmen.

Persönliche Utensilien der Patienten werden nicht auf dem Waschbeckenrand oder in Spritznähe, sondern möglichst spritzgeschützt oder in ausreichendem Abstand zum Waschbecken aufbewahrt.

Waschbecken mit rückwärts in der Wandung gelegenen Abflussöffnungen reduzieren die Umgebungskontamination. Bei Neu- oder Umbaumaßnahmen wird Waschbecken mit nachgewiesener geringerer Umgebungskontamination der Vorzug gegeben.

Es gelten die Empfehlungen in der KRINKO-Empfehlung Händehygiene in Einrichtungen des Gesundheitswesens zu den Anforderungen eines Handwaschplatzes.

Waschbecken bestehen aus Materialien, die leicht zu reinigen und mit Präparaten auf der Basis von Per- bzw. Chlorverbindungen zu desinfizieren sind, über glatte Oberflächen verfügen und keine Fissuren aufweisen.

Der störungsfreie Ablauf von Waschbecken ist regelmäßig zu Der störungsfreie Ablauf von Waschbecken ist in kurzen Abständen zu kontrollieren, z. B. vom Reinigungspersonal. kontrollieren.

Bei Verstopfung bzw. Rückstau sind die Ursachen durch in Hygiene geschultes technisches Personal zu beseitigen und hiernach eine desinfizierende Reinigung mit Perverbindungen bzw. Chlor vorzunehmen. Das Hygienefachpersonal wird informiert.

Waschbecken sind in ausreichendem Abstand (mind. $1 \mathrm{~m}$ ) zum Patientenbett zu positionieren.

Bei Neuplanung ist auf Waschbecken im Patientenzimmer zu verzichten, wobei in diesem Fall sicherzustellen ist, dass dem medizinischen Personal in der näheren Umgebung Handwaschplätze für eine eventuell erforderliche Händewaschung zur Verfügung stehen (z. B. bei Betreuung von Patienten mit Clostridioides difficile).

Es kann in Erwägung gezogen werden, die Abflussleitungen der Waschbecken mit thermisch desinfizierenden Siphonsystemen auszustatten.

In Bereichen mit mobilen Patienten (z. B. Hämato-Onkologie) haben die Patientenzimmer eigene, direkt vom Zimmer aus erreichbare Nasszellen.

Keine besonderen Bei Neu- und Umbauten sind Anforderungen
Spülrandfreien Toilettenschüsseln ist der Vorzug zu geben. spülrandfreie Toilettenschüssel zu empfehlen.

Nicht gestattet nach DIN EN 1717 sind Intimwasserspülung (Hygieneduschen) über Schlauchverbindungen mit direktem Anschluss an einen Wasserhahn.

Während der Toilettenspülung wird der Toilettendeckel geschlossen. Darüber werden Patienten z. B. durch Hinweisschilder im Sanitärbereich informiert.

Bei Verstopfung bzw. Rückstau sind die Ursachen durch in Hygiene geschultes technisches Personal zu beseitigen und hiernach eine desinfizierende Reinigung mit Perverbindungen bzw. Chlor vorzunehmen. Das Hygienefachpersonal wird informiert. 


\begin{tabular}{llll} 
Tab. 2 (Fortsetzung) & $\begin{array}{l}\text { Bereich ohne } \\
\text { Infektionsrisiko (I) }\end{array}$ & $\begin{array}{l}\text { Bereich mit möglichem } \\
\text { Infektionsrisiko (II) }\end{array}$ & Bereich mit besonderem Infektionsrisiko (III) \\
\hline Steckbeckenspüler & $\begin{array}{l}\text { Keine besonderen } \\
\text { Anforderungen }\end{array}$ & $\begin{array}{l}\text { Steckbeckenspüler werden bei rein thermischen Verfahren mit einem A0-Wert von mindes- } \\
\text { tens } 600 \text { betrieben. } \\
\text { Bei der Neu- und Umbauplanung wird auf eine ausreichende Anzahl von Steckbeckenspülern } \\
\text { in strategisch sinnvollen Bereichen der Station geachtet. }\end{array}$ \\
& - & $\begin{array}{l}\text { In Bereichen für bestimmungsgemäß infektiöse bzW. sehr } \\
\text { schwer immunsupprimierte Patienten ist das Vorhan- } \\
\text { densein von Steckbeckenspülern sinnvoll, die jeweils } \\
\text { den Zimmern zugeordnet sind (bei Um- und Neubau } \\
\text { beachten). }\end{array}$
\end{tabular}

\section{Duschen und Duschbecken}
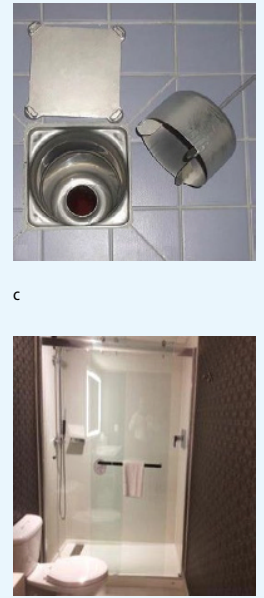

Schmetterlingsmücken (Clogmia albipunctata)

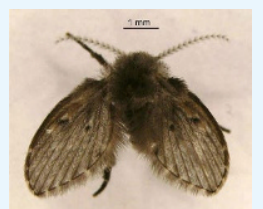

Keine besonderen Anforderungen

Keine besonderen Anforderungen

Bei Neu- und Umbauplanungen ist vorzusehen, dass Duschbecken und deren Abfluss gegenüber Reinigungs- und Desinfektionsmitteln beständig sind (inkl. Desinfektion mit Perverbindungen bzw. Halogenen).

Speziell konstruierte Abflussöffnungen (Abwasserkappen) erlauben die Desinfektion. Bei Neu- oder Umbaumaßnahmen können diese in Erwägung gezogen werden.

Bei neu eingerichteten Duschen wird der Abfluss nicht im direkten Positionsbereich des Duschenden positioniert; wandnahe Bodenabläufe sind zu bevorzugen. Bei bodengleichen Duschen ohne Spritzschutz sollte das Bodengefälle zum Fußbodenabfluss von der Tür bis zum Abfluss vorhanden sein, um stehendes Wasser zu verhindern.

Insbesondere bei Flachduschen wird baulich-funktionell immer auf einen ausreichenden Abstand zwischen Abflussöffnung und Abwasserrohr geachtet. Abwasser darf nicht sichtbar in den Duschabläufen stehen, da die Abwasserleitung Zimmer untereinander ohne Barriere verbindet.

Bei Verstopfung bzw. Rückstau sind die Ursachen durch in Hygiene geschultes technisches Personal zu beseitigen und hiernach eine desinfizierende Reinigung mit Perverbindungen bzw. Chlor vorzunehmen. Das Hygienefachpersonal wird informiert.

Auf Duschvorhänge wird in der Regel verzichtet.

Kann auf Duschvorhänge nicht verzichtet werden, werden diese mit einem hinreichenden Abstand zum Fußboden bzw. zur Duschtasse installiert. Regelungen zu regelmäßiger Aufbereitung bzw. zum Austausch von Duschvorhängen werden im Hygieneplan festgelegt.

Das Auftreten von Schmetterlingsmücken in Sanitärbereichen der Patienten oder in Küchen ist dem Hygienefachpersonal zu melden. 


\section{Tab. 2 (Fortsetzung)}

\begin{tabular}{ll}
\hline Regelungsbereich & $\begin{array}{l}\text { Bereich ohne } \\
\text { Infektionsrisiko (I) }\end{array}$ \\
\hline $\begin{array}{l}\text { Ausgussbecken in unreinen } \\
\text { Räumen }\end{array}$ & $\begin{array}{l}\text { Keine besonderen } \\
\text { Anforderungen }\end{array}$ \\
\hline
\end{tabular}

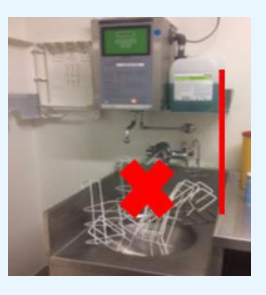

b

Desinfektion und Reinigung

\section{Abwasserleitung}

Maßnahmen bei Havariefällen von Abwasserleitungen

Zentrale Küchen in medizinischen Einrichtungen

\section{Bereich mit möglichem Bereich mit besonderem Infektionsrisiko (III)}

Infektionsrisiko (II)

Ausgussbecken sind räumlich bzw. durch Spritzschutz von reinen Bereichen separiert, sofern nicht eine räumliche Trennung ermöglicht werden kann.

Desinfektionsmittel-Dosiergeräte (rein) werden bei Um- und Neubaumaßnahmen nicht über Ausgussbecken (unrein), in die kontaminierte Flüssigkeiten entsorgt werden, installiert.

Innerhalb des Ausgussbeckens gibt es keine Ringspülung, die einer direkten desinfizierenden Reinigung nicht zugänglich ist.' Bei Tätigkeit am Ausgussbecken mit potentiell kontaminierten Flüssigkeiten wird persönliche Schutzausrüstung getragen (Schürze, Schutzhandschuhe), die anschließend unmittelbar abzulegen und zu entsorgen ist.

Keine besonderen Anforderungen

Waschbecken, Abflussöffnungen, Duschbecken und Toiletten werden bevorzugt mit Desinfektionsmittelpräparaten auf Peroxid- oder Chlorbasis desinfiziert. Der mechanischen Reinigung kommt eine entscheidende Funktion zu.

Baulich-funktionell verfügen Abwasserleitungen entsprechend DIN 1986 über eine ausreichende Neigung zur Vermeidung von Stagnation.

Das Hygienefachpersonal ist über Havariefälle in abwasserführenden Systemen mit Rückstau in den Patientenbereich zu informieren, um geeignete flankierende Schutzmaßnahmen u. a. zur Desinfektion festzulegen und die Durchführung zu überwachen. Näheres ist im Hygieneplan zu regeln.

Beim Einsatz von Reinigungsspiralen und anderen Verfahren zur Behebung von Verstopfungen im Abwassersystem wird auf den Schutz der Umgebung und der Kleidung des Durchführenden vor einer fäkalen Kontamination geachtet. Es erfolgt ein geschützter Abtransport kontaminierter Utensilien. Hiernach ist eine umfassende Desinfektion der Umgebung durchzuführen.

- $\quad$ Bei der Abklärung der Ursache und Behebung von Verstopfungen ist das Hygienefachpersonal einzubeziehen, sofern dafür Tätigkeiten in Patientennähe erforderlich sind.

Reinigungs- und Desinfektionsverfahren im Bereich der Böden und Abflüsse werden hinsichtlich der Kontaminationsgefahr einer Risikobewertung unterzogen.

Bei der Verwendung von Hochdruckreinigungsgeräten besteht die Gefahr der Verbreitung von Bakterien vom Fußboden und aus Abflüssen in höher gelegene Arbeitsbereiche. Dem Scheuer-Wischverfahren zur desinfizierenden Reinigung wird der Vorzug gegeben.

Ein betroffener Küchenbereich wird nach Wartungs- und Reparaturarbeiten am Abwassersystem vollständig gereinigt und mit einem geeigneten Desinfektionsmittel desinfiziert. Geräte und Hilfsmittel, die für Wartungsarbeiten im Abwassersystem der Krankenhausküchen benötigt werden, sind möglichst nur dort zum Einsatz zu bringen.

a Nachgedruckt von Journal of Hospital Infection, Vol. 102, Issue 1, Arenega-Bou et al., Carbapenem-resistant Enterobacteriaceae dispersal from sinks is linked to drain position and drainage rates in a laboratory model system", Seiten 63-69, Copyright 2019, mit freundlicher Genehmigung von @ Elsevier

${ }^{\mathrm{b}}$ Nachgedruckt mit freundlicher Genehmigung von (c) Herrn Prof. Dr. Exner

c Nachgedruckt mit freundlicher Genehmigung von (c) Frau Dr. Kossow

${ }^{\mathrm{d}}$ Nachgedruckt mit freundlicher Genehmigung von (c) Herrn Prof. Dr. Faulde

'In den im Ausgussbecken befindlichen Ringspülungen können gramnegative Bakterien persistieren. Sie sind einer Wischdesinfektion nur schwer zugänglich. 